\title{
A MARKOV ADDITIVE RISK PROCESS WITH A DIVIDEND BARRIER
}

\author{
ESTHER FROSTIG, ${ }^{*}$ University of Haifa
}

\begin{abstract}
We study a risk process with dividend barrier $b$ where the claims arrive according to a Markovian additive process (MAP). For spectrally negative MAPs, we present linear equations for the expected discounted dividends and the expected discounted penalty function. We apply results for the first exit times of spectrally negative Lévy processes and change-of-measure techniques. Explicit expressions are given when there are positive and negative claims, with phase-type distribution.
\end{abstract}

Keywords: Markov arrival process; spectrally negative Lévy process; phase-type distribution; exit time; reflected process

2010 Mathematics Subject Classification: Primary 62P05; 60G51; 60J27; 60J75;

$60 \mathrm{G} 46 ; 60 \mathrm{~J} 55$

\section{Introduction}

Risk models evolving in a Markovian environment have received considerable attention in recent years. In these models the environmental state is a continuous-time Markov chain (CTMC) with a finite state space. When the environment state is $i$, the process behaves as a compound Poisson risk model, where the claim amount distribution, the premium rate, and the claim arrival intensity depend on $i$. The process can be perturbed by a Brownian motion with state-dependent volatility. In addition, a claim arrival can occur with a transition of the random environment states. Such models are called Markov additive risk processes. In this paper we study a Markov additive risk process with a dividend barrier, where all the surplus above a given barrier is paid as dividends to the shareholders.

Ahn and Badescu (2007) considered a Markov additive risk process without the Brownian motion component. They applied the fluid version of the model, and the matrix-analytic approach for the first passage times. For the same model, Li and Lu (2007), (2008), and Lu and $\mathrm{Li}$ (2009) derived integrodifferential equations for the moments of the discounted dividends and the penalty function. Lu and Tsai (2007) considered a similar model with a Brownian motion component and obtained an integrodifferential equation for the expected discounted penalty function. More recently, Cheung and Landriault (2009) considered a model discussed in this paper. They derived integrodifferential equations for the quantities of interest and solved them using Laplace transforms. The Laplace transforms can be easily converted when the claim amounts have rational transforms.

Our method is different. We consider a Markov additive risk process with phase-type claim amount. We use the semiregenerative points in the process to obtain linear equations for the expected discounted dividends and the expected discounted penalty function. Then we apply the change of measure and the fluid version of the process to solve these equations explicitly.

Received 9 August 2011; revision received 28 August 2012.

* Postal address: Department of Statistics, University of Haifa, Haifa 31905, Israel.

Email address: frostig@stat.haifa.ac.il

Research supported by the Israel Science Foundation, under grant 606/09. 
Our penalty function is less general than in Cheung and Landriault (2009) since it is only a function of the time to ruin and the deficit at ruin, but not of the surplus before ruin as in Cheung and Landriault. We obtain the expected discounted dividends while Cheung and Landriault obtained higher moments of the discounted dividends. However, applying the methods presented in this paper, we obtain some significant generalizations of the results of Cheung and Landriault (2009). Cheung and Landriault assumed that at each environmental state the risk process is perturbed by a Brownian motion, i.e. the volatility coefficient is positive for all the environmental states, and the premium rate is positive for all states. Using the method presented in this paper, we obtain results for risk processes with positive or negative premium rate, and positive or zero volatility coefficient (depending on the random environment).

The main advantage of our method is that it can be applied to both negative and positive claims. Positive claims are usually considered as losses or claim payments, while negative claims (or positive jumps) are lump gains. Avanzi et al. (2007) and Avanzi and Gerber (2008) studied risk models with negative claims only, referring to them as dual models, which are a special case of ours. In recent years there has been a growing interest in models with two-sided jumps. Recently, Cheung (2011) obtained differential equations for a very general risk model with two-sided jumps.

Note that Cheung and Landriault's results apply to general claim amount distributions, and their results have a simple form when the claim amounts have rational transforms. Our results are applicable to phase-type claim amounts. However, the phase-type distribution is dense in the class of distributions of nonnegative random variables; thus, we can apply our results to general distributions. It seems that Cheung and Landriault's results are more applicable when the premium rates and the volatility coefficients are positive for all states and there are only negative jumps.

Cheung and Landriault (2009) also considered a Markov additive risk process, where the barrier strategy depends on the environmental state, i.e. there are dividend payments at state $i$ only if the surplus is above $b_{i}$. They obtained the moments of the discounted dividends for the two-state case and described how to generalize it for an arbitrary number of states. We apply our methods to analyzing the two-state case for positive claims (only) and for the dual model, i.e. negative claims (only). We obtain linear equations for the expected discounted dividends and the expected discounted penalty function. These equations are very easily solved-their coefficients are the solutions of a system of linear equations.

The rest of the paper is organized as follows. In Section 2 we introduce some properties of Lévy processes, and review basic properties of Markov additive processes (MAPs) that are used later. In Section 3 we explain the general idea of our method, and how to obtain the expected discounted dividends and the expected discounted time to ruin. In Section 4 we present explicit results for positive claims with phase-type distribution. In Section 5 we extend the results to the case where there are both positive and negative claims. We prove that the dividends paid until ruin have phase-type distribution and find its parameters. A numerical example is given in Section 6. Finally, in Section 7 we consider the state-dependent barrier strategy, where the claims are either positive or negative.

\section{Preliminaries}

\subsection{Lévy process}

In this paper we consider only the Lévy risk process $X(t)$ which is the sum of two independent processes. The first is a Brownian motion with drift and the second is a compound Poisson 
process:

$$
X(t)=\mu t-\sum_{j=1}^{N(t)} Z_{j}+\sigma B(t), \quad \sigma \geq 0 .
$$

Here $N(t)$ is a Poisson process with rate $\lambda$, and the $Z_{j}$ are independent, identically distributed (i.i.d.) random variables with distribution $G$ and moment generating function $\hat{G}(s)=$ $\int_{-\infty}^{\infty} \mathrm{e}^{s x} \mathrm{~d} G(x)$. Note that in the classical risk process the $Z_{j}$ are nonnegative random variables, i.e. positive claims. The Lévy exponent $\varphi(\theta)$ for this process is given by

$$
\varphi(\theta)=\log \left(\mathbb{E}\left[\mathrm{e}^{\theta X(1)}\right]\right)=\theta \mu+\frac{1}{2} \sigma^{2} \theta^{2}-\lambda(1-\hat{G}(-\theta)) .
$$

An important tool to analyze a Lévy risk process is the Wald martingale (see Asmussen (2003, Remark 8.9, p. 104)). Let

$$
L(\theta, t)=\exp (\theta X(t)-\varphi(\theta) t) .
$$

Let $\mathcal{F}_{t}$ denote the natural filtration for $X(t)$, let $I(A)$ denote the indicator of the event $A$, and let $\mathbb{P}(A)$ denote the probability of event $A$. Define

$$
\mathbb{P}^{c}(A)=\mathbb{E}[L(c, t) I(A)] ;
$$

$\mathbb{P}^{c}$ is a probability measure. Under $\mathbb{P}^{c}, X(t)$ is a Lévy process with Lévy exponent

$$
\varphi_{c}(\theta)=\varphi(\theta+c)-\varphi(c) .
$$

In our case,

$$
\varphi_{c}(\theta)=\theta\left(\mu+\sigma^{2} c\right)+\frac{\sigma^{2} \theta^{2}}{2}-\lambda \hat{G}(-c)\left(1-\frac{\hat{G}(-(\theta+c))}{\hat{G}(-c)}\right) .
$$

Thus, under the change of measure, $X(t)$ is the sum of a Brownian motion with drift $\mu+c \sigma^{2}$, and a compound Poisson process with arrival rate $\lambda \hat{G}(-c)$ and jump distribution $\tilde{G}$, where

$$
\mathrm{d} \tilde{G}(x)=\frac{\mathrm{e}^{-c x} \mathrm{~d} G(x)}{\hat{G}(-c)} .
$$

For more details on the change of measure, we refer the reader to Chapter XIII.3 of Asmussen (2003). A Lévy process without positive jumps is known as a spectrally negative Lévy process. Let $\Phi(q)$ be the largest root of the equation $\varphi(\theta)=q$, which exists (by convexity of $\varphi(\theta)$ ) with $\Phi(q) \geq 0$. Let $\tau_{b}^{+}=\inf \{t>0: X(t) \geq b\}$ and $\tau_{a}^{-}=\inf \{t>0: X(t) \leq a\}$.

Definition 2.1. Let $X(t)$ be a spectrally negative Lévy process. For $q \geq 0$, there exists a unique continuous function $W^{(q)}:[0, \infty] \rightarrow[0, \infty]$, called the $q$-scale function, such that

$$
\int_{0}^{\infty} \mathrm{e}^{-\theta x} W^{(q)}(x) \mathrm{d} x=\frac{1}{\varphi(\theta)-q}, \quad \theta>\Phi(q) .
$$

Another function related to the $q$-scale function is the adjoint $q$-scale function $Z^{(q)}$.

Definition 2.2. For $q \geq 0$,

$$
Z^{(q)}(x)=1+q \int_{0}^{x} W^{(q)}(s) \mathrm{d} s .
$$


The importance of the functions $W^{(q)}(x)$ and $Z^{(q)}$ is due to the following proposition.

Proposition 2.1. Let $X(t)$ be a spectrally negative Lévy process. Then, for $0<x<b$ and $q \geq 0$,

$$
\begin{gathered}
\mathbb{E}_{x}\left[\mathrm{e}^{-q \tau_{b}^{+}} I\left(\tau_{b}^{+}<\tau_{0}^{-}\right)\right]=\frac{W^{(q)}(x)}{W^{(q)}(b)}, \\
\mathbb{E}_{x}\left[\mathrm{e}^{-q \tau_{0}^{-}} I\left(\tau_{b}^{+}>\tau_{0}^{-}\right)\right]=Z^{(q)}(x)-W^{(q)}(x) \frac{Z^{(q)}(b)}{W^{(q)}(b)} .
\end{gathered}
$$

The scale function of a Brownian motion with drift $\mu$ and coefficient of variation $\sigma>0$ is

$$
W^{(q)}(x)=\frac{2}{\sqrt{2 q \sigma^{2}+\mu^{2}}} \mathrm{e}^{-\mu x / \sigma^{2}} \sinh \left(\frac{x}{\sigma^{2}} \sqrt{2 q \sigma^{2}+\mu^{2}}\right) .
$$

The scale function for the case $\sigma=0$ is

$$
W^{(q)}(x)=\frac{1}{\mu} \mathrm{e}^{q x / \mu} .
$$

For more details on the scale function and the first passage time of Lévy processes, see Chapter 8 of Kyprianou (2006) and the references therein. In the sequel we will also consider the reflected Lévy process. Consider a spectrally negative Lévy process $X(t), X(0)=0$. Let

$$
M(t)=\sup (s \leq t: X(s)),
$$

and let

$$
Y(t)=M(t)-X(t)
$$

$Y(t)$ is the reflected process at its maximum. Let $T_{b}=\inf \{t: Y(t) \geq b\}$ be the first time that $Y(t)$ reaches $b$. The Laplace transform of $T_{b}$ is given by Proposition 2 of Pistorius (2004):

$$
\mathcal{L}_{T_{b}}(q)=\mathbb{E}_{0}\left[\mathrm{e}^{-q T_{b}}\right]=Z^{(q)}(b)-\frac{q\left(W^{(q)}(b)\right)^{2}}{W^{(q)^{\prime}}(b)} .
$$

Denote by $\varepsilon_{q}$ an independent, exponentially distributed random variable with parameter $q$. Let $\delta>0$, and let

$$
q r_{q}(x) \mathrm{d} x=\mathbb{E}\left[\mathrm{e}^{-\delta \varepsilon_{q}} I\left(\varepsilon_{q}<T_{b}, Y\left(\varepsilon_{q}\right) \in \mathrm{d} x\right)\right] .
$$

Then, by Theorem 1 of Pistorius (2004),

$$
\begin{aligned}
& r_{q}(y)=W^{(q+\delta)}(b) \frac{W^{(\delta+q)^{\prime}}(y)}{W^{(\delta+q)^{\prime}}(b)}-W^{(\delta+q)}(y), \quad y \neq 0, \\
& r_{q}(0)=W^{(q+\delta)}(b) \frac{W^{(q)}(0)}{W^{(q)^{\prime}}(b)} .
\end{aligned}
$$

Let $x \wedge y=\min (x, y)$.

Theorem 2.1. It holds that

$$
\mathbb{E} \int_{0}^{T_{b} \wedge \varepsilon_{q}} \mathrm{e}^{-\delta t} \mathrm{~d} M(t)=\frac{W^{(q+\delta)}(b)}{W^{(q+\delta)^{\prime}}(b)} .
$$

The proof of Theorem 2.1 for the case where the upper limit of the integral is $T_{b}$ is the same as that of Theorem 1 of Avram et al. (2004), and uses excursion theory. The proof for the case where the limit is as in the theorem is straightforward. 


\subsection{Markov additive arrival process}

A barrier free Markov additive risk process $\{R(t), t \geq 0\}$ is defined in terms of the background CTMP $J(t)$ on a finite state space $E$ with $n$ states, and transition intensity matrix $\boldsymbol{\Lambda}$. When $J(t)=i$, the risk process behaves as a compound Poisson process perturbed by a Brownian motion. Thus, claims arrive according to a Poisson process $N_{i}(t)$, at rate $\beta_{i}$, and are i.i.d. with distribution $G_{i i}$, density function $g_{i i}$, and moment generating function (MGF) $\hat{G}_{i i}(\theta)=\mathbb{E}\left[\mathrm{e}^{\theta Z_{i}}\right]$. Denote the premium rate by $c_{i}$. The process might be perturbed by a Brownian motion $B(t)$, with volatility coefficient $\sigma_{i}$. Thus, when $J(t)=i$, the risk process behaves as a Lévy process with Lévy exponent

$$
\varphi_{i}(\alpha)=\log \mathbb{E}\left[\mathrm{e}^{\theta R_{i}(1)}\right]=c_{i} \alpha+\frac{1}{2} \sigma_{i}^{2} \alpha^{2}-\beta_{i}\left(1-\hat{G}_{i i}(-\alpha)\right) .
$$

Note that, when $\sigma_{i}=0$, the process increases or decreases linearly between jumps.

Additional claim arrivals might occur upon transition of $J(t)$ from $i$ to $j$ : with probability $p_{i j}$, a claim arrival occurs upon transition from state $i$ to state $j$. This claim has distribution $G_{i j}$, density function $g_{i j}$, and MGF $\hat{G}_{i j}(\theta)=\int_{-\infty}^{\infty} \mathrm{e}^{\theta x} G_{i j}(\mathrm{~d} x), i \neq j$. In the classical risk process the claims are positive; thus, only negative jumps are considered. Later in the paper we consider both positive and negative jumps. We decompose the transition rate matrix $\boldsymbol{\Lambda}$ as

$$
\boldsymbol{\Lambda}=\boldsymbol{D}_{0}+\boldsymbol{D}_{1}
$$

where $\boldsymbol{D}_{0}$ is the transition rate matrix without arrivals and $\boldsymbol{D}_{1}$ is the transition rate matrix with claim arrivals:

$$
\begin{gathered}
D_{0}(i, j)=\Lambda(i, j)\left(1-p_{i j}\right), \quad i \neq j, \quad D_{0}(i, i)=\Lambda(i, i)-\beta_{i}, \\
D_{1}(i, j)=\Lambda(i, j) p_{i j}, \quad i \neq j, \quad D_{1}(i, i)=\beta_{i} .
\end{gathered}
$$

Note that $\sigma_{i}$ can be also 0. As in Breuer (2008), (2010), we exclude the case in which $c_{i}=$ $\sigma_{i}=0$. In our model $c_{i}$ can be either positive or negative. As in Breuer (2008), (2010), we decompose the state space $E$ as $E=E_{p} \cup E_{n} \cup E_{\sigma}$, where

$$
\begin{aligned}
& E_{p}=\left\{i \in E, c_{i}>0, \sigma_{i}=0\right\}, \\
& E_{n}=\left\{i \in E, c_{i}<0, \sigma_{i}=0\right\}, \\
& E_{\sigma}=\left\{i \in E, \sigma_{i}>0\right\} .
\end{aligned}
$$

Let $|A|$ be the number of elements in a finite set $A$, and let $n_{p}=\left|E_{p}\right|, n_{n}=\left|E_{n}\right|$, and $n_{\sigma}=\left|E_{\sigma}\right|$.

Let $\hat{\boldsymbol{D}}(\alpha)$ be a matrix with $(i, j)$ th element, $i \neq j$, equal to $\lambda_{i j} p_{i j}\left(\hat{G}_{i j}(-\alpha)-1\right)$ and $(i, i)$ th element equal to 0 . Define the cumulant generating function matrix of $R(t)$ as

$$
\boldsymbol{K}(\alpha)=\boldsymbol{\Lambda}+\operatorname{diag}\left(\varphi_{1}(\alpha), \ldots, \varphi_{n}(\alpha)\right)+\hat{\boldsymbol{D}}(\alpha) .
$$

Proposition 2.2 of Asmussen (2003, Chapter XI) states that $\mathbb{E}\left[\mathrm{e}^{\alpha R(t)} ; J(t)=j \mid J(0)=i\right]$ is the $(i, j)$ th element of the matrix $\mathrm{e}^{\boldsymbol{K}(\alpha) t}$. The matrix $\boldsymbol{K}(\alpha)$ has a real eigenvalue $\kappa(\alpha)$ with maximal real part. Let the column vector $\boldsymbol{h}(\alpha)$ be its right eigenvector. Its components may be chosen to be positive. When $\alpha=0$, the components of $\boldsymbol{h}(0)$ are equal and can be chosen to be 1 . The eigenvalue $\kappa(\alpha)$ is convex in $\alpha$. The derivative of $\kappa(\alpha)$ at 0 determines the asymptotic behavior of $R(t)$ at $\infty$. Denote by $\Phi(q)$ the right inverse of $\kappa(\alpha)$ :

$$
\Phi(q)=\sup \{\alpha \geq 0: \kappa(\alpha)=q\} .
$$


The properties of $\kappa$ imply that $\Phi(q)>0$ for $q>0$. We have $\Phi(0)>0$ if and only if $\kappa^{\prime}(0)<0$; otherwise, $\Phi(0)=0$. For more details, see Chapter XI of Asmussen (2003). In the sequel we will apply the Wald martingale for the process $R(t)$.

\subsubsection{Wald martingale and change of measure.}

Proposition 2.2. (Asmussen (2003, Chapter XI).) It holds that

$$
L(t)=\mathrm{e}^{\alpha R(t)-\kappa(\alpha) t} h_{J(t)}(\alpha)
$$

is a martingale.

Let $\mathcal{F}$ be the natural filtration, and let $\mathbb{P}$ be the probability measure defined by the MAP $R(t)$. Let $A \in \mathscr{F}_{t}$. Define the probability measure by $\mathbb{P}^{\alpha}(A)=\mathbb{E}[L(t) I(A)]$. By Theorem 8.2 of Asmussen (2003, Chapter XIII), the probability $\mathbb{P}^{\alpha}$ defines a MAP with the following parameters. The transition rate matrix $\boldsymbol{\Lambda}^{\alpha}$ is given by

$$
\boldsymbol{\Lambda}^{\alpha}=\boldsymbol{\Delta}_{\boldsymbol{h}(\alpha)}^{-1} \boldsymbol{K}(\alpha) \boldsymbol{\Delta}_{\boldsymbol{h}(\alpha)}-\kappa(\alpha) \boldsymbol{I},
$$

where $\boldsymbol{\Delta}_{\boldsymbol{h}(\alpha)}$ is a diagonal matrix with $h_{i}(\alpha)$ on the diagonal, and $\boldsymbol{I}$ is an $n \times n$ identity matrix. The $(i, j)$ th element of $\boldsymbol{\Lambda}^{\alpha}$ is

$$
\Lambda^{\alpha}(i, j)=\frac{h_{j}(\alpha)}{h_{i}(\alpha)} \lambda(i, j)\left[1+p_{i j}\left(\hat{G}_{i j}(-\alpha)-1\right)\right], \quad i \neq j .
$$

The probability for a claim triggered by a transition from $i$ to $j$ is

$$
p_{i j}^{\alpha}=\frac{p_{i j} \hat{G}_{i j}(-\alpha)}{1+p_{i j}\left(\hat{G}_{i j}(-\alpha)-1\right)} .
$$

The distribution of the claim arriving at state $i$ or upon transition from state $i$ to $j$ is

$$
G_{i j}^{\alpha}(\mathrm{d} x)=\frac{\mathrm{e}^{-\alpha x} G_{i j}(\mathrm{~d} x)}{\hat{G}_{i j}(-\alpha)} .
$$

The drift parameter is $c_{i}^{\alpha}=c_{i}+\sigma_{i}^{2} \alpha$, while $\sigma_{i}^{\alpha}=\sigma_{i}$. The claim arrival intensity when $J(t)=i$ is $\beta_{i}^{\alpha}=\beta_{i} \hat{G}_{i i}(-\alpha)$. Note that $\boldsymbol{K}^{\alpha}(\theta)=\boldsymbol{\Delta}_{\boldsymbol{h}(\alpha)}^{-1} \boldsymbol{K}(\alpha+\theta) \boldsymbol{\Delta}_{\boldsymbol{h}(\alpha)}-\kappa(\alpha) \boldsymbol{I}$.

Throughout the paper, we denote by $\mathbb{E}_{i, u}$ and $\mathbb{P}_{i, u}$ the conditional expectation and probability given that $R_{b}(0)=u$ and $J(0)=i$.

2.2.2. Exit times for MAPs. Let $\tau_{b}^{+}=\inf \{t>0: R(t) \geq b\}$ and $\tau_{a}^{-}=\inf \{t>0: R(t) \leq a\}$. Similarly to Proposition 2.1 for spectrally negative Lévy processes, Kyprianou and Palmowski (2008) proved the following theorem for the spectrally negative MAP. We cite only the parts of this theorem relevant to our paper.

Theorem 2.2. For each $q \geq 0$, there exist $n \times n$ matrix functions $\boldsymbol{W}^{(q)}(\cdot)$ and $\boldsymbol{Z}^{(q)}(\cdot)$ such that the following statements hold (for convenience, we will write $\boldsymbol{W}^{(0)}=\boldsymbol{W}$ ).

(i) For $x \leq b$, the $(i, j)$ th element of

$$
\boldsymbol{W}^{(q)}(x) \boldsymbol{W}^{(q)}(b)^{-1}
$$

is

$$
\mathbb{E}_{i, x}\left[\mathrm{e}^{-q \tau_{b}^{+}} I\left(\tau_{b}^{+}<\tau_{0}^{-}\right) ; J\left(\tau_{b}^{+}=j\right)\right]
$$


(ii) The $(i, j)$ th element of

$$
\boldsymbol{Z}^{(q)}(x)-\boldsymbol{W}^{(q)}(x) \boldsymbol{W}^{(q)}(b)^{-1} \boldsymbol{Z}^{(q)}(b)
$$

is

$$
\mathbb{E}_{i, x}\left[\mathrm{e}^{-q \tau_{0}^{-}} I\left(\tau_{0}^{-}<\tau_{b}^{+}\right) ; J\left(\tau_{0}^{-}=j\right)\right]
$$

\section{The general spectrally negative Markov additive risk process}

\subsection{The expected discounted dividends}

In this section we consider a Markov additive risk process $R(t)$ with only negative jumps (positive claims) and a dividend barrier $b$. Denote by $R_{b}(t)=R(t)-D(t)$, where $D(t)$ is the amount of dividends paid until time $t$. Let $V_{i}(u, b)$ be the expected discounted dividends paid until ruin when the initial modulating state is $i$, the initial reserve is $u, u \leq b$, and the discount factor is $\delta$ :

$$
\left.V_{i}(u, b)=\sum_{j \in E} \mathbb{E}_{i, u}\left[\mathrm{e}^{-\delta \tau_{b}^{+}}, \tau_{b}^{+}<\tau_{0}^{-}, J\left(\tau_{b}^{+}\right)=j\right)\right] V_{j}(b, b) .
$$

Given that $J(0)=i$, let $\mathcal{E}_{i}$ be the time until a claim arrival or change of the modulating state. Here $\varepsilon_{i}$ is an exponentially distributed random variable with parameter $\eta_{i}=\beta_{i}-\Lambda(i, i)$, and is independent of the risk process. Given that $R_{b}(0)=b$, then up to time $\mathcal{E}_{i}$ the process $b-R_{b}(t)$ behaves as a process $Y_{i}$, which is the reflection at the maximum of the Lévy process $X_{i}(t)=c_{i} t+\sigma_{i} B(t)$. Let $T_{b, i}$ be the time until the reflected process $Y_{i}$ reaches the level $b$. The Laplace transform of $T_{b, i}$ follows by substituting the scale function of $X_{i}$ in (2.3). We calculate $V_{i}(b, b)$ in two steps.

1. Calculate $w_{i}$, the expected discounted dividends up to time $\varepsilon_{i} \wedge T_{b, i}$.

2. Calculate the expected discounted dividends from time $\varepsilon_{i} \wedge T_{b, i}$ until ruin.

Let $r_{i}(x)=r_{\eta_{i}}(x)$ be as defined in (2.4) and (2.5) with $q$ replaced by $\eta_{i}$, and where the scale function in (2.5) is the scale function for $X_{i}(t)$, given by (2.1) or (2.2).

Proposition 3.1. Let $V_{i}(b, b)$ be the expected discounted dividends given that $J(0)=i$ and $R_{b}(0)=b$. Then, for $i=1, \ldots, n$,

$$
\begin{aligned}
V_{i}(b, b)= & w_{i} \\
& +\sum_{j=1}^{n} \sum_{k=1}^{n} D_{1}(i, k) \int_{0}^{b} \int_{0}^{x} r_{i}(x-y) G_{i k}(\mathrm{~d} y) \\
& \quad \times \mathbb{E}_{k, b-x}\left[\mathrm{e}^{-\delta \tau_{b}^{+}}, \tau_{b}^{+}<\tau_{0}^{-}, J\left(\tau_{b}^{+}\right)=j\right] \mathrm{d} x V_{j}(b, b) \\
& +\sum_{j=1}^{m} \sum_{k \neq i} D_{0}(i, k) \int_{0}^{b} r_{i}(x) \mathbb{E}_{k, b-x}\left[\mathrm{e}^{-\delta \tau_{b}^{+}}, \tau_{b}^{+}<\tau_{0}^{-}, J\left(\tau_{b}^{+}\right)=j\right] \mathrm{d} x V_{j}(b, b)
\end{aligned}
$$

The proof is straightforward from the definition of $r_{i}$ and the fact that the process is semiregenerative: semiregenerative points are when the process reaches $b$. Note that

(i) $\mathbb{E}_{i, b-x}\left[\mathrm{e}^{-\delta \tau_{b}^{+}}, \tau_{b}^{+}<\tau_{0}^{-}, J\left(\tau_{b}^{+}\right)=j\right]$ is the $(i, j)$ th element of $\boldsymbol{W}^{(\delta)}(b-x) \boldsymbol{W}^{(\delta)}(b)^{-1}$ given in Theorem 2.2, 
(ii) $w_{i}$ is the expected discounted dividends up to time $\varepsilon_{i} \wedge T_{b, i}$, given that $R_{b}(0)=b$. Let

$$
M_{i}(t)=\sup _{0 \leq s \leq t}\left(c_{i} s+\sigma_{i} B(s)\right)
$$

Following Avram et al. (2004), and applying Theorem 2.1 and (2.1), we obtain, for $\sigma_{i}>0$,

$$
w_{i}=\mathbb{E} \int_{0}^{\mathcal{E}_{i} \wedge T_{b, i}} \mathrm{e}^{-\delta t} \mathrm{~d} M_{i}(t)=\frac{W^{\left(\eta_{i}+\delta\right)}(b)}{W^{\left(\eta_{i}+\delta\right)^{\prime}}(b)}=\left(\frac{-c_{i}}{\sigma_{i}^{2}}+\frac{a_{i}}{\sigma_{i}^{2}} \operatorname{coth}\left(\frac{b}{\sigma_{i}^{2} a_{i}}\right)\right)^{-1},
$$

where

$$
a_{i}=\sqrt{c_{i}+2 \sigma_{i}^{2}\left(\lambda_{i}+\beta_{i}+\delta\right)}
$$

For $\sigma_{i}=0$ and $c_{i}>0$, we obtain

$$
w_{i}=\frac{c_{i}}{\delta+\eta_{i}}
$$

The main difficulty lies in deriving $\boldsymbol{W}^{(q)}(x) \boldsymbol{W}^{(q)}(b)^{-1}$. In the next section we will show how to obtain an explicit solution when the claim amount is phase type.

\subsection{The expected discounted time to ruin}

Let $\mathcal{T}_{b}$ be the time to ruin, i.e. the first time that $R_{b}(t)$ drops below 0 , and let $\xi_{0}^{-}$be the deficit at ruin. Let $f$ be a function called the penalty function, and let $\omega_{i}(u, b)$ be its expected discounted value:

$$
\omega_{i}(u, b)=\mathbb{E}_{i, u}\left[\mathrm{e}^{-\delta \mathcal{T}_{b}} f\left(\xi_{0}^{-}\right)\right] .
$$

In this subsection we present similar equations to (3.2) for the Laplace transform of the time to ruin, i.e. for $\omega_{i}(u, b)$ for the special case where $f(\cdot)=1$. Thus, our goal in this section is to obtain

$$
\omega_{i}(u, b)=\mathbb{E}_{u, i}\left[\mathrm{e}^{-\delta \mathcal{T}_{b}}\right] .
$$

For $0<u<b$,

$$
\omega_{i}(u, b)=\mathbb{E}_{i, u}\left[\mathrm{e}^{-\delta \tau_{0}^{-}}, \tau_{0}^{-}<\tau_{b}^{+}\right]+\sum_{j \in E} \mathbb{E}_{i, u}\left[\mathrm{e}^{-\delta \tau_{b}^{+}}, \tau_{b}^{+}<\tau_{0}^{-}, J\left(\tau_{b}^{+}\right)=j\right] \omega_{j}(b, b) .
$$

We can form a system of linear equations for $\omega_{i}(b, b)$ :

$$
\begin{aligned}
\omega_{i}(b, b)= & \mathbb{E}\left[\mathrm{e}^{-\delta T_{b, i}} I\left(T_{b, i}<\mathcal{E}_{i}\right)\right]+\sum_{k=1}^{n} D_{1}(i, k) \int_{0}^{b} r_{i}(x)\left(1-G_{i k}(b-x)\right) \mathrm{d} x \\
& +\sum_{j=1}^{n} \sum_{k=1}^{n} D_{1}(i, k) \int_{0}^{b} \int_{0}^{x} r_{i}(x-y) G_{i k}(\mathrm{~d} y) \\
& \times \mathbb{E}_{k, b-x}\left[\mathrm{e}^{-\delta \tau_{0}^{-}}, \tau_{0}^{-}<\tau_{b}^{+}, J\left(\tau_{0}^{-}\right)=j\right] \mathrm{d} x \\
& +\sum_{j=1}^{n} \sum_{k=1}^{n} D_{0}(i, k) \int_{0}^{b} r_{i}(x) \mathbb{E}_{k, b-x}\left[\mathrm{e}^{-\delta \tau_{0}^{-}}, \tau_{0}^{-}<\tau_{b}^{+}, J\left(\tau_{0}^{-}\right)=j\right] \mathrm{d} x \\
& +\sum_{j=1}^{n} \sum_{k=1}^{n} D_{1}(i, k) \int_{0}^{b} \int_{0}^{x} r_{i}(x-y) G_{i, k}(\mathrm{~d} y) \\
& \times \mathbb{E}_{k, b-x}\left[\mathrm{e}^{-\delta \tau_{b}^{+}}, \tau_{b}^{+}<\tau_{0}^{-}, J\left(\tau_{b}^{+}\right)=j\right] \mathrm{d} x \omega_{j}(b, b)
\end{aligned}
$$




$$
\begin{aligned}
& +\sum_{j=1}^{m} \sum_{k \neq i} D_{0}(i, k) \\
& \quad \times \int_{0}^{b} r_{i}(x) \mathbb{E}_{k, b-x}\left[\mathrm{e}^{-\delta \tau_{b}^{+}}, \tau_{b}^{+}<\tau_{0}^{-}, J\left(\tau_{b}^{+}\right)=j\right] \mathrm{d} x \omega_{j}(b, b) .
\end{aligned}
$$

The first term in (3.6) is the expected time to ruin occurring by diffusion before $\mathcal{E}_{i}$. The second term in (3.6) describes the expected discounted time to ruin, when ruin occurs at time $\mathcal{E}_{i}$ due to a claim arrival with a state transition. The terms in (3.7) and (3.8) are the expected discounted times to ruin when ruin occurs after $\mathcal{E}_{i}$ and before reaching $b$. The terms in (3.9) and (3.10) describe the expected discounted ruin times, where ruin occurs after $\varepsilon_{i}$ and after the surplus reaches $b$. Note that $\mathbb{E}_{k, b-x}\left[\mathrm{e}^{-\delta \tau_{0}^{-}}, \tau_{0}^{-}<\tau_{b}^{+}, J\left(\tau_{0}^{-}\right)=j\right]$ is the $(k, j)$ th element of the matrix in (2.7) at $b-x$, while $\mathbb{E}_{k, b-x}\left[\mathrm{e}^{-\delta \tau_{b}^{+}}, \tau_{b}^{+}<\tau_{0}^{-}, J\left(\tau_{b}^{+}\right)=j\right]$ is the $(k, j)$ th element of the matrix in (2.6).

In the next section we assume that the claim amounts are phase type, allowing us to obtain explicit $\omega_{i}(u, b)$.

\section{Spectrally negative risk process with phase-type claim amount}

In this section we assume that the claim size distributions $G_{i j}$ are phase type with representation $\left(\boldsymbol{\pi}_{i j}, \boldsymbol{T}_{i j}\right)$. Thus, $G_{i j}$ is the distribution of the time until absorption of a CTMC with $m_{i j}$ transient states and one absorbing state. Let $\boldsymbol{T}_{i j}$ be its intensity transition matrix among the transient states, let $\boldsymbol{\pi}_{i j}$ be the vector of the initial probabilities, and let $\boldsymbol{t}_{i j}=-\boldsymbol{T}_{i j} \mathbf{1}$ be the intensity rate vector to absorption from each state, where $\mathbf{1}$ is a column vector of $1 \mathrm{~s}$ with the appropriate dimension. We have

$$
1-G_{i j}(x)=\pi_{i j} \exp \left(\boldsymbol{T}_{i j} x\right) \mathbf{1}, \quad x>0 .
$$

The density function is $g_{i j}(x)=\pi_{i j} \exp \left(\boldsymbol{T}_{i j} x\right) \boldsymbol{t}_{i j}$.

To derive (3.2) for the expected discounted dividends, and (3.7)-(3.8) for the expected discounted penalty function, we need to find expressions for the expected discounted time to reach the level $b$ before ruin, and the expected discounted time to reach 0 before $b$. Let

$$
v_{k, j}^{+}(x)=\mathbb{E}_{k, x}\left[\mathrm{e}^{-\delta \tau_{b}^{+}}, \tau_{b}^{+}<\tau_{0}^{-}, J\left(\tau_{b}^{+}\right)=j\right] .
$$

Let $\Phi(\delta)>0$ be a number such that the eigenvalue with the maximal real part of $\boldsymbol{K}(\Phi(\delta))$ is $\delta$, and let $\boldsymbol{h}(\Phi(\delta))$ be the right eigenvector for $\kappa(\Phi(\alpha))$. Applying the change-of-measure formula given in Equation (17) of Kyprianou and Palmowski (2008) or Asmussen (2003, p. 377), we obtain

$$
\begin{aligned}
v_{k, j}^{+}(x) & =\mathbb{E}_{k, x}\left[\mathrm{e}^{-\delta \tau_{b}^{+}}, \tau_{b}^{+}<\tau_{0}^{-}, J\left(\tau_{b}^{+}\right)=j\right] \\
& =h_{k}(\Phi(\delta)) \mathbb{P}_{k, x}^{\Phi(\delta)}\left(\tau_{b}^{+}<\tau_{0}^{-}, J\left(\tau_{b}^{+}\right)=j\right) \frac{1}{h_{j}(\Phi(\delta))} \mathrm{e}^{-\Phi(\delta)(b-x)} .
\end{aligned}
$$

Thus, the problem is reduced to finding the probability of hitting $b$ before 0 under the measure $\mathbb{P}^{\Phi(\delta)}$. Under the measure $\mathbb{P}^{\Phi(\delta)}$, the process $R(t)$ is a MAP. We denote its parameters with a tilde. Asmussen (2003, Chapter XIII.8) obtained the following parameters for the process under $\mathbb{P}^{\Phi(\delta)}$.

(P1) Premium rate $\tilde{c}_{i}=c_{i}+\Phi(\delta) \sigma_{i}^{2}$.

(P2) The volatility, $\tilde{\sigma}_{i}^{2}=\sigma_{i}^{2}$. 
(P3) The claim arrival rate when $J(t)=i$ is $\tilde{\beta}_{i}=\beta_{i} \hat{G}_{i i}(-\Phi(\delta))$.

(P4) The transition rates

$$
\tilde{\Lambda}(i, j)=\frac{h_{j}(\Phi(\delta))}{h_{i}(\Phi(\delta))} \Lambda(i, j)\left[1+p_{i j}\left(\hat{G}_{i j}(-\Phi(\delta))-1\right)\right] .
$$

(P5) The probability of claim at transition from state $i$ to state $j$,

$$
\tilde{p}_{i j}=\frac{p_{i j} \hat{G}_{i j}(-\Phi(\delta))}{1+p_{i j}\left(\hat{G}_{i j}(-\Phi(\delta))-1\right)} .
$$

(P6) The claim distribution at transition from state $i$ to $j$,

$$
\tilde{G}_{i j}(\mathrm{~d} x)=\frac{\mathrm{e}^{-\Phi(\delta) x}}{\hat{G}_{i j}(-\Phi(\delta))} G_{i j}(\mathrm{~d} x) .
$$

(P7) $\tilde{\boldsymbol{K}}(\alpha)=\boldsymbol{\Delta}_{\boldsymbol{h}(\Phi(\delta))}^{-1} \boldsymbol{K}(\Phi(\delta)+\alpha) \boldsymbol{\Delta}_{\boldsymbol{h}(\Phi(\delta))}-\delta \boldsymbol{I}$.

Let

$$
\boldsymbol{k}_{i j}(\theta)=\left(-\theta \boldsymbol{I}-\boldsymbol{T}_{i j}\right)^{-1} \boldsymbol{t}_{i j},
$$

and let $\boldsymbol{\Delta}_{\boldsymbol{k}_{i j}(\theta)}$ be a diagonal matrix with the components of $\boldsymbol{k}_{i j}(\theta)$ on the diagonal. We will need the following result obtained by Asmussen (1989).

Lemma 4.1. The claim distribution $\tilde{G}_{i j}$ is phase type with representation $\left(\tilde{\boldsymbol{\pi}}_{i j}, \tilde{\boldsymbol{T}}_{i j}\right)$, where

$$
\begin{gathered}
\tilde{\boldsymbol{T}}=\boldsymbol{\Delta}_{\boldsymbol{k}_{i j}(-\Phi(\delta))}^{-1} \boldsymbol{T}_{i j} \boldsymbol{\Delta}_{\boldsymbol{k}_{i j}(-\Phi(\delta))}-\Phi(\delta) \boldsymbol{I}, \\
\tilde{\boldsymbol{\pi}}_{i j}=\boldsymbol{\pi}_{i j} \boldsymbol{\Delta}_{\boldsymbol{k}_{i j}(-\Phi(\delta))} / \hat{G}(-\Phi(\delta)), \\
\tilde{\boldsymbol{t}}_{i j}=\boldsymbol{\Delta}_{\boldsymbol{k}_{i j}(-\Phi(\delta))}^{-1} \boldsymbol{t}_{i j} .
\end{gathered}
$$

To obtain $v_{i j}^{+}(x)$ in (4.2), we need to derive $\gamma_{i j}^{+}(x)=\mathbb{P}_{k, x}^{\Phi(\delta)}\left(\tau_{b}^{+}<\tau_{0}^{-}, J\left(\tau_{b}^{+}\right)=j\right)$. To find $\gamma_{i j}^{+}(x)$, we consider the fluid model of the process $R(t)$, under the measure $\mathbb{P}^{\Phi(\delta)}$. In the fluid model a downwards jump of size $v$ is replaced by a linear line with slope -1 for $v$ time units. Thus, when in state $i \in E$, the process evolves as a Brownian motion with drift $\tilde{c}_{i}$, or linearly with slope $\tilde{c}_{i}$, and in states $(i j, l)$ linearly with slope -1 . We augment the state space $E$ of $J$ by $E_{-}=\left\{(i j, k), i, j \in E, k=1, \ldots, m_{i j}\right\}$, where $(i k, j)$ denotes the $i k$ th claim at phase $j$.

The fluid model behaves as a MAP without jumps, and state space $E \cup E_{-}=$ $E_{p} \cup E_{n} \cup E_{\sigma} \cup E_{-}$. The transition rate matrix $\boldsymbol{Q}$ for the fluid model under the measure $\mathbb{P}^{\Phi(\delta)}$ is

$$
Q(i, j)= \begin{cases}\tilde{\Lambda}(i, j)\left(1-\tilde{p}_{i j}\right), & i \neq j, i, j \in E, \\ \tilde{\Lambda}(i, i)-\tilde{\beta}_{i}, & i=j, i \in E, \\ \tilde{\beta}_{i} \tilde{\pi}_{i i}(k), & i \in E, j=(i i, k), \\ \tilde{\Lambda}(i, k) \tilde{p}_{i k} \tilde{\pi}_{i k}(l), & i, k \in E, j=(i k, l), \\ \tilde{T}_{s t}(r, l), & i=(s t, r), j=(s t, l), \\ \tilde{t}_{s t}(l), & i=(s t, l), j=t .\end{cases}
$$

Let $\left(R^{f}(t), J^{f}(t)\right)$ be the MAP that describes the risk fluid model, where $R^{f}$ is the fluid level and $J^{f}$ is the modulated state. Then

$$
\mathbb{E}^{\Phi(\delta)}\left[\mathrm{e}^{\alpha R^{f}(t)}, J^{f}(t)=\tilde{j} \mid R^{f}(0)=0, J^{f}(0)=\tilde{i}\right], \quad \tilde{i}, \tilde{j} \in E \cup E_{-},
$$


is the $(\tilde{i}, \tilde{j})$ th element of $\mathrm{e}^{\boldsymbol{F}(\alpha) t}$, where

$$
\boldsymbol{F}(\alpha)=\boldsymbol{Q}+\operatorname{diag}\left(\varphi_{1}(\alpha), \ldots, \varphi_{n}(\alpha),-\alpha, \ldots,-\alpha\right)
$$

and $\varphi_{i}(\alpha)=\tilde{c}_{i} \alpha+\sigma_{i}^{2} \alpha^{2} / 2$ is the cumulant matrix for the fluid model. Let $\tau_{b}^{+f}$ and $\tau_{0}^{-f}$ be the hitting times for the fluid model at levels $b$ and 0 , respectively, i.e. $\tau_{b}^{f+}=\min \left\{t: R^{f}(t)=b\right\}$ and $\tau_{0}^{f-}=\min \left\{t: R^{f}(t)=0\right\}$. It is not difficult to see that the probability that $R^{f}$ hits $b$ before it hits 0 is the same as the probability of the same event for the original process (under the measure $\left.\mathbb{P}^{\Phi(\delta)}\right)$ :

$$
\mathbb{P}_{i, x}^{\Phi(\delta)}\left(\tau_{b}^{+f}<\tau_{0}^{-f}, J\left(\tau_{b}^{+f}\right)=j\right)=\mathbb{P}_{i, x}^{\Phi(\delta)}\left(\tau_{b}^{+}<\tau_{0}^{-}, J\left(\tau_{b}^{+}\right)=j\right) .
$$

Consider now the fluid model under the probability measure $\mathbb{P}^{\Phi(\delta)}$. For $i, j \in E$, let $\gamma_{i, j}^{+}(x)$ be the probability of hitting $b$ before hitting 0 with $J^{f}\left(\tau_{b}^{f+}\right)=j$, let $\gamma_{i, j}^{-}(x)$ be the probability of hitting 0 before hitting $b$ with $J^{f}\left(\tau_{0}^{f-}\right)=j \in E$, and let $\gamma_{i,(k j, l)}^{-}(x)$ be the probability of hitting 0 before hitting $b$ with $J^{f}\left(\tau_{0}^{f-}\right)=(k j, l), k, j \in E$. Thus,

$$
\begin{aligned}
\gamma_{k, j}^{+}(x) & =\mathbb{P}^{\Phi(\delta)}\left(J^{f}\left(\tau_{b}^{+}\right)=j, \tau_{b}^{+}<\tau_{0}^{-} \mid R_{b}(0)=x, J(0)=k\right), \quad k, j \in E, \\
\gamma_{k, j}^{-}(x) & =\mathbb{P}^{\Phi(\delta)}\left(J^{f}\left(\tau_{0}^{-}\right)=j, \tau_{0}^{-}<\tau_{b}^{+} \mid R_{b}(0)=x, J(0)=k\right), \quad k, j \in E, \\
\gamma_{k,(i j, l)}^{-}(x) & =\mathbb{P}^{\Phi(\delta)}\left(J^{f}\left(\tau_{0}^{-}\right)=(i j, l), \tau_{0}^{-}<\tau_{b}^{+} \mid R_{b}(0)=x, J(0)=k\right), \quad i, k, j \in E .
\end{aligned}
$$

To find the above probabilities, we apply the multidimensional Wald martingale as follows. Let $|\boldsymbol{F}(\alpha)|$ be the determinant of $\boldsymbol{F}(\alpha)$. Here $|\boldsymbol{F}(\alpha)|$ is a polynomial of degree $N=n_{p}+$ $n_{n}+2 n_{\sigma}+\sum_{i=1}^{n} \sum_{j=1}^{n} m_{i j}$. Assume that the equation $|\boldsymbol{F}(\alpha)|=0$ has $N$ different roots $\alpha_{1}, \ldots, \alpha_{N}$. Clearly, one of the roots, say $\alpha_{1}$, is 0 . The eigenvalue of $\boldsymbol{F}\left(\alpha_{i}\right)$ is 0 , i.e. $\kappa\left(\alpha_{i}\right)=0$. Let the $\boldsymbol{h}^{f}\left(\alpha_{i}\right)$ be the corresponding right eigenvectors. We can choose $\boldsymbol{h}^{f}\left(\alpha_{1}\right)=\boldsymbol{h}(0)$ to be equal to 1 .

By Proposition 2.2, the process $\left\{\mathrm{e}^{\alpha_{k}\left(R^{f}(t)\right.} h_{J(t)}^{f}\left(\alpha_{k}\right)\right\}$ is a martingale. Let $R^{f}(0)=x$, $0<x<b$, and $J^{f}(0)=k$.

Applying the optional sampling theorem for this martingale and for the stopping time $\min \left\{\tau_{b}^{+f}, \tau_{0}^{-f}\right\}$, we obtain, for $k \in E$,

$$
\begin{aligned}
\mathrm{e}^{\alpha_{r} x} h_{k}^{f}\left(\alpha_{r}\right)= & \mathrm{e}^{\alpha_{r} b} \sum_{j \in E_{\sigma} \cup E_{p}} \gamma_{k j}^{+}(x) h_{j}^{f}\left(\alpha_{r}\right)+\sum_{j \in E_{\sigma} \cup E_{n}} \gamma_{k j}^{-}(x) h_{j}^{f}\left(\alpha_{r}\right) \\
& +\sum_{j=1}^{n} \sum_{l=1}^{n} \sum_{o=1}^{m_{j l}} \gamma_{k,(j l, o)}^{-}(x) h_{(j l, o)}^{f}\left(\alpha_{r}\right) .
\end{aligned}
$$

Remark 4.1. Note that $\gamma_{k,(j l, o)}^{-}(x)$ is the probability under $\mathbb{P}^{\Phi(\delta)}$ that, given $R(0)=x$, $J(0)=k$, ruin occurs before reaching the dividend barrier by a $j l$ th claim at phase $o$. Similarly, $\gamma_{k j}^{-}(x)$ is the probability of ruin by diffusion before reaching the dividend barrier.

Define

$$
\begin{aligned}
A_{i, k, j} & =\int_{0}^{b} r_{i}(x) v_{k, j}^{+}(b-x) \mathrm{d} x, \quad i \neq k, \\
A c_{i, k, j} & =\int_{0}^{b} \int_{y=0}^{x} r_{i}(y) g_{i k}(x-y) \mathrm{d} y v_{k, j}^{+}(b-x) \mathrm{d} x, \quad i \neq k .
\end{aligned}
$$

Assume that $R(0)=b$ and $J(0)=i$. The quantities $A_{i, k, j}$ and $A c_{i, k, j}$ are the discounted times to reach level $b$ when the modulated state is $j$, before ruin and after $\varepsilon_{i}$, when the transition at $\varepsilon_{i}$ is respectively without or with a claim arrival. 


\subsection{The expected discounted dividends}

The $V_{i}(b, b)$ satisfy

$$
V_{i}(b, b)=w_{i}+\sum_{j=1}^{n}\left(\sum_{k=1}^{n} A c_{i, k, j} D_{1}(i, k)+\sum_{k \neq i} A_{i, k, j} D_{0}(i, k)\right) V_{j}(b, b) .
$$

By (3.1), for $u<b$,

$$
V_{i}(u, b)=\sum_{j \in E} v_{i, j}^{+}(u) V_{j}(b, b) .
$$

\subsection{The expected discounted penalty function}

Equation (3.5) can be written as

$$
\begin{aligned}
\omega_{i}(u, b)= & \sum_{j \in E} \mathbb{E}_{i, u}\left[\mathrm{e}^{-\delta \tau_{0}^{-}} f\left(\xi_{0}^{-}\right), \tau_{0}^{-}<\tau_{b}^{+}, J\left(\tau_{0}^{-}\right)=j\right] \\
& +\sum_{j \in E} \mathbb{E}_{i, u}\left[\mathrm{e}^{-\delta \tau_{b}^{+}}, \tau_{b}^{+}<\tau_{0}^{-}, J\left(\tau_{b}^{+}\right)=j\right] \omega_{j}(b, b) .
\end{aligned}
$$

Applying the change-of-measure formula, we obtain

$$
\begin{aligned}
v_{i, j}^{-}(u, 0) & =\mathbb{E}_{i, u}\left[\mathrm{e}^{-\delta \tau_{0}^{-}}, \xi_{0}^{-}=0, \tau_{0}^{-}<\tau_{b}^{+}, J\left(\tau_{0}^{-}\right)=j\right] \\
& =h_{i}(\Phi(\delta)) \gamma_{i, j}^{-}(u) \frac{1}{h_{j}(\Phi(\delta))} \mathrm{e}^{\Phi(\delta) u},
\end{aligned}
$$

where $\gamma_{i, j}^{-}(u)$ is given by solving (4.4). Let

$$
\begin{aligned}
v_{i, j}^{-}(u, z) \mathrm{d} z & =\mathbb{E}_{i, u}\left[\mathrm{e}^{-\delta \tau_{0}^{-}}, \xi_{0}^{-} \in \mathrm{d} z, \tau_{0}^{-}<\tau_{b}^{+}, J\left(\tau_{0}^{-}\right)=j\right] \\
& =h_{i}(\Phi(\delta)) \mathbb{P}_{i, u}^{\Phi(\delta)}\left(\xi_{0}^{-} \in \mathrm{d} z, \tau_{0}^{-}<\tau_{b}^{+}, J\left(\tau_{0}^{-}\right)=j\right) \frac{1}{h_{j}(\Phi(\delta))} \mathrm{e}^{\Phi(\delta)(u+z)} .
\end{aligned}
$$

Note that the deficit at ruin can be positive if and only if ruin is caused by a claim arrival in some state $k$ or with a state transition from state $k$ to $j$. Let $\boldsymbol{\gamma}_{i, k j}^{-}(u)$ be a row vector of dimension $m_{k j}$, with $\gamma_{i, k j}^{-}(u)=\left(\gamma_{i,(k j, 1)}^{-}(u), \ldots, \gamma_{i,\left(k j, m_{k j}\right)}^{-}(u)\right)$, where the $\gamma_{i,(k j, l)}^{-}(u)$ are obtained by solving (4.4). Under the measure $\mathbb{P}^{\Phi(\delta)}$, the deficit at ruin is phase type, specifically,

$$
\mathbb{P}_{i, u}^{\Phi(\delta)}\left(\xi_{0}^{-} \in \mathrm{d} z, \tau_{0}^{-}<\tau_{b}^{+}, J\left(\tau_{0}^{-}\right)=j\right)=\sum_{k \in E} \boldsymbol{\gamma}_{i, k j}^{-}(u) \exp \left(\tilde{\boldsymbol{T}}_{k j} z\right) \tilde{\boldsymbol{t}}_{k j} \mathrm{~d} z .
$$

Applying (4.9) yields

$$
v_{i, j}^{-}(u, z)=h_{i}(\Phi(\delta))\left(\sum_{k \in E} \boldsymbol{\gamma}_{i, k j}^{-}(u) \exp \left(\tilde{\boldsymbol{T}}_{k j} z\right) \tilde{\boldsymbol{t}}_{k j}\right) \frac{1}{h_{j}(\Phi(\delta))} \mathrm{e}^{\Phi(\delta)(u+z)} .
$$

By (4.8) and (4.10), we find that the expected discounted penalty function when the initial reserve is $u, J(0)=i$, and ruin occurs before reaching $b$ is given by

$$
\begin{aligned}
\omega_{0, i}(u, b) & \left.=\sum_{j \in E} \mathbb{E}_{i, u}\left[\mathrm{e}^{-\delta \tau_{0}^{-}} f\left(\xi_{0}^{-}\right), \tau_{0}^{-}<\tau_{b}^{+}, J\left(\tau_{0}^{-}\right)=j\right)\right] \\
& =\sum_{j \in E} v_{i, j}^{-}(u, 0) f(0)+\sum_{j \in E} \int_{z=0}^{\infty} v_{i, j}^{-}(u, z) f(z) \mathrm{d} z .
\end{aligned}
$$


Consider now $\omega_{i}(b, b)$, the expected discounted penalty function when the initial reserve is $b$ and the initial modulating state is $i$. First consider the case where $\min \left(\mathcal{E}_{i}, T_{b, i}\right)=T_{b, i}$. In this case ruin occurs by diffusion, and the expected discounted penalty function is given by

$$
\omega_{1, i}(b)=f(0) \int_{0}^{\infty} \mathrm{d} F_{T_{b, i}}(t) \exp \left(-\left(\eta_{i}+\delta\right) t\right)=f(0) \mathcal{L}_{T_{b, i}}\left(\eta_{i}+\delta\right),
$$

where $\mathcal{L}_{T_{b}, i}(\theta)$ is obtained by substituting (2.1) or (2.2) into (2.3).

We now present the system of linear equations for $\omega_{i}(b, b)$.

Proposition 4.1. We have

$$
\begin{aligned}
\omega_{i}(b, b)= & \omega_{1, i}(b)+\sum_{k \in E} D_{1}(i, k) \int_{x=0}^{b} \int_{z=0}^{\infty} r_{i}(x) g_{i k}(b-x+z) f(z) \mathrm{d} z \mathrm{~d} x \\
& +\sum_{k \in E} D_{1}(i, k) \int_{x=0}^{b} \int_{y=0}^{x} r_{i}(y) g_{i k}(x-y) \mathrm{d} y \omega_{0, k}(b-x, b) \mathrm{d} x \\
& +\sum_{k \in E, k \neq i} D_{0}(i, k) \int_{x=0}^{b} r_{i}(x) \omega_{0, k}(b-x, b) \mathrm{d} x \\
& +\sum_{j \in E} \sum_{k \in E} D_{1}(i, k) A c_{i, k, j} \omega_{j}(b, b) \\
& +\sum_{j \in E} \sum_{k \in E} D_{0}(i, k) A_{i, k, j} \omega_{j}(b, b) .
\end{aligned}
$$

Proof. The term $\omega_{1, i}(b)$ is the discounted penalty function when ruin occurs due to a diffusion when $T_{b, i}<\mathcal{E}_{i}$. Assume that $\varepsilon_{i}<T_{b, i}$. The second term in (4.13) is the discounted penalty function when ruin occurs at time $\varepsilon_{i}$ due to a claim arrival. The terms in (4.14) and (4.15) describe the expected penalty functions when ruin occurs after time $\varepsilon_{i}$, before the surplus reaches level $b$, where the state transition at $\varepsilon_{i}$ occurs with or without a claim arrival. The terms in (4.16) and (4.17) are the discounted penalty functions when the level $b$ is reached before ruin after $\varepsilon_{i}$, where the state transition at $\varepsilon_{i}$ occurs with or without a claim arrival.

For $f(z)=1$, we obtain the Laplace transform of the time to ruin. For $f(z)=\mathrm{e}^{-\theta z}$, we obtain the joint Laplace transform of the time to ruin and the deficit at ruin.

\section{Markov additive risk process with positive and negative claims}

In this section we extend the results to a Markov additive risk process with positive and negative claims, i.e. negative and positive jumps. When $J(t)=i$, positive claims arrive according to a Poisson process at rate $\beta_{i}^{-}$, and negative claims (upward jumps) arrive according to a Poisson process at rate $\beta_{i}^{+}$. The distribution of the positive claim is $G_{i i}^{-}$and its density is $g_{i i}^{-}$. The distribution of the negative claim is $G_{i i}^{+}$and its density $g_{i i}^{+}$. A transition of the modulated state from $i$ to $j$ is accompanied by a positive claim with probability $p_{i, j}^{-}$, claim distribution $G_{i j}^{-}$, and density $g_{i j}^{-}$. A transition of the modulated state from $i$ to $j$ is accompanied by a negative claim with probability $p_{i, j}^{+}$, claim distribution $G_{i j}^{+}$, and density $g_{i j}^{+}$. With probability $1-p_{i, j}^{+}-p_{i, j}^{-}$, there is no arrival upon a state transition. Denote by $\boldsymbol{D}_{0}$ the transition rate matrix without arrivals; thus, $D_{0}(i, i)=\Lambda(i, i)-\beta_{i}^{+}-\beta_{i}^{-}$and $D_{0}(i, j)=\Lambda(i, j)\left(1-p_{i, j}^{+}-p_{i, j}^{-}\right), i \neq j$. 
Similarly, let $D_{1}^{ \pm}(i, j)=\Lambda(i, j) p_{i, j}^{ \pm}, i \neq j$, and $D_{1}^{ \pm}(i, i)=\beta_{i}^{ \pm}$. In this case, for $i \neq j$, the $(i, j)$ th element of $\hat{\boldsymbol{D}}(\alpha)$ is $\Lambda_{i j} p_{i j}^{+}\left(\hat{G}_{i j}^{+}(\alpha)-1\right)+\Lambda_{i j} p_{i j}^{-}\left(\hat{G}_{i j}^{-}(-\alpha)-1\right)$.

The distribution functions $G_{i j}^{ \pm}$are phase type with representation $\left(\boldsymbol{\pi}_{i j}^{ \pm}, \boldsymbol{T}_{i j}^{ \pm}\right)$, with $m_{i j}^{ \pm}$states, where $\boldsymbol{t}_{i j}^{ \pm}$is the transition rate vector to the absorbing state. Denote the phases of the positive claims (downward jumps) by $l^{-}$, and the phases of the negative claims (upward jumps) by $l^{+}$. Note that, when a positive jumps crosses the dividend barrier $b$, all the overflow above $b$ is paid as dividends. The $(i, j)$ th element of $\mathrm{e}^{t \boldsymbol{K}(\alpha)}$ is $\mathbb{E}_{i, 0}\left[\mathrm{e}^{\alpha R(t)}, J(t)=j\right]$, where

$$
\boldsymbol{K}(\alpha)=\boldsymbol{\Lambda}+\operatorname{diag}\left(\varphi_{1}(\alpha), \ldots, \varphi_{n}(\alpha)\right)+\hat{\boldsymbol{D}}(\alpha)
$$

and

$$
\varphi_{i}(\alpha)=c_{i} \alpha+\frac{1}{2} \sigma_{i}^{2} \alpha^{2}+\left(\beta_{i}^{+}\left(\hat{G}_{i i}^{+}(\alpha)-1\right)+\beta_{i}^{-}\left(\hat{G}_{i i}^{-}(-\alpha)-1\right)\right)
$$

Let $\Phi(\delta)$ and $\kappa(\alpha)$ be as described in Section 2.2. Under the probability measure $\mathrm{P}^{\Phi(\delta)}$, the process behaves as a MAP with parameters (P1)-(P7). Let $\boldsymbol{k}_{i j}^{+}(\theta)=\left(-\theta \boldsymbol{I}-\boldsymbol{T}_{i j}^{+}\right)^{-1} \boldsymbol{t}_{i j}^{+}$ and $\boldsymbol{k}_{i j}^{-}(\theta)=\left(\theta \boldsymbol{I}-\boldsymbol{T}_{i j}^{-}\right)^{-1} \boldsymbol{t}_{i j}^{-}$. Let $\boldsymbol{\Delta}_{\boldsymbol{k}_{i j}^{ \pm}(\Phi(\delta))}$ be a diagonal matrix with the components of $\boldsymbol{k}_{i j}^{ \pm}(\Phi(\delta))$ on the diagonal. The distribution functions $\tilde{G}_{i j}^{ \pm}$are phase type with representation $\left(\tilde{\boldsymbol{\pi}}_{i j}^{ \pm}, \tilde{\boldsymbol{T}}_{i j}^{ \pm}\right)$as described in Lemma 4.1.

Let $\xi_{b}^{+}$be the overflow above $b$ when the surplus hits the level $b$. We have $\xi_{b}^{+}>0$ if $R(t)$ hits $b$ by a negative claim, and $\xi_{b}^{+}=0$ if $R(t)$ hits $b$ by diffusion. Similarly, let $\xi_{0}^{-}$be the deficit at ruin. We have $\xi_{0}^{-}>0$ if ruin occurs due to a positive claim, and $\xi_{0}^{-}=0$ if ruin occurs due to a diffusion. To obtain the expected discounted dividends and the expected discounted penalty function, we need the following expectations:

$$
\begin{aligned}
v_{i, j}^{+}(u, z) \mathrm{d} z & =\mathbb{E}_{i, u}\left[\mathrm{e}^{-\delta \tau_{b}^{+}} I\left(\tau_{b}^{+}<\tau_{0}^{-}\right), \xi_{b}^{+} \in \mathrm{d} z, J\left(\tau_{b}^{+}\right)=j\right], \\
v_{i, j}^{+}(u, 0) & =\mathbb{E}_{i, u}\left[\mathrm{e}^{-\delta \tau_{b}^{+}} I\left(\tau_{b}^{+}<\tau_{0}^{-}\right), \xi_{b}^{+}=0, J\left(\tau_{b}^{+}\right)=j\right], \\
v_{i, j}^{-}(u, z) \mathrm{d} z & =\mathbb{E}_{i, u}\left[\mathrm{e}^{-\delta \tau_{0}^{-}} I\left(\tau_{0}^{-}<\tau_{b}^{+}\right), \xi_{0}^{-} \in \mathrm{d} z, J\left(\tau_{0}^{-}\right)=j\right], \\
v_{i, j}^{-}(u, 0) & =\mathbb{E}_{i, u}\left[\mathrm{e}^{-\delta \tau_{0}^{-}} I\left(\tau_{0}^{-}<\tau_{b}^{+}\right), \xi_{0}^{-}=0, J\left(\tau_{0}^{-}\right)=j\right] .
\end{aligned}
$$

Applying the change-of-measure formula as in (4.2), we obtain

$$
\begin{aligned}
v_{i, j}^{+}(u, z) \mathrm{d} z= & h_{i}(\Phi(\delta)) \mathbb{P}_{i, u}^{\Phi(\delta)}\left(\tau_{b}^{+}<\tau_{0}^{-}, J\left(\tau_{b}^{+}\right)=j, \xi_{b}^{+} \in \mathrm{d} z\right) \\
& \times\left(h_{j}(\Phi(\delta))\right)^{-1} \mathrm{e}^{-\Phi(\delta)(b-u+z)}, \\
v_{i, j}^{+}(u, 0)= & h_{i}(\Phi(\delta)) \mathbb{P}_{i, u}^{\Phi(\delta)}\left(\tau_{b}^{+}<\tau_{0}^{-}, J\left(\tau_{b}^{+}\right)=j, \xi_{b}^{+}=0\right) \\
& \times\left(h_{j}(\Phi(\delta))\right)^{-1} \mathrm{e}^{-\Phi(\delta)(b-u)}, \\
v_{i, j}^{-}(u, z) \mathrm{d} z= & h_{i}(\Phi(\delta)) \mathbb{P}_{i, u}^{\Phi(\delta)}\left(\tau_{0}^{-}<\tau_{b}^{+}, J\left(\tau_{0}^{-}\right)=j, \xi_{0}^{-} \in \mathrm{d} z\right) \\
& \times\left(h_{j}(\Phi(\delta))\right)^{-1} \mathrm{e}^{\Phi(\delta)(u+z)}, \\
v_{i, j}^{-}(u, 0)= & h_{i}(\Phi(\delta)) \mathbb{P}_{i, u}^{\Phi(\delta)}\left(\tau_{0}^{-}<\tau_{b}^{+}, J\left(\tau_{0}^{-}\right)=j, \xi_{0}^{-}=0\right) \\
& \times\left(h_{j}(\Phi(\delta))\right)^{-1} \mathrm{e}^{u \Phi(\delta)} .
\end{aligned}
$$


Thus, to obtain (5.1), we need to derive

$$
\begin{aligned}
\gamma_{i, j}^{+}(u, z) \mathrm{d} z & =\mathbb{P}_{i, u}^{\Phi(\delta)}\left(\tau_{b}^{+}<\tau_{0}^{-}, J\left(\tau_{b}^{+}\right)=j, \xi_{b}^{+} \in \mathrm{d} z\right), \\
\gamma_{i, j}^{+}(u, 0) & =\mathbb{P}_{i, u}^{\Phi(\delta)}\left(\tau_{b}^{+}<\tau_{0}^{-}, J\left(\tau_{b}^{+}\right)=j, \xi_{b}^{+}=0\right), \\
\gamma^{-}(u, z) \mathrm{d} z & =\mathbb{P}_{i, u}^{\Phi(\delta)}\left(\tau_{0}^{-}<\tau_{b}^{+}, J\left(\tau_{0}^{-}\right)=j, \xi_{0}^{-} \in \mathrm{d} z\right), \\
\gamma_{i, j}^{-}(u, 0) & =\mathbb{P}_{i, u}^{\Phi(\delta)}\left(\tau_{0}^{-}<\tau_{b}^{+}, J\left(\tau_{0}^{-}\right)=j, \xi_{0}^{-}=0\right) .
\end{aligned}
$$

To derive the terms in (5.2), we consider the fluid version of the model under $\mathbb{P}^{\Phi(\delta)}$. In this model negative jumps are replaced by a line with slope -1 and positive jumps with a line with slope 1 . The fluid model is a MAP without jumps with state space $E \cup E_{-} \cup E_{+}$, where $E_{ \pm}=\left\{\left(i j, l^{ \pm}\right), i, j, \in E\right\}$. Let $\boldsymbol{Q}$ be its transition rate matrix under $\mathbb{P}^{\Phi}$, defined by

$$
Q(i, j)= \begin{cases}\tilde{\Lambda}(i, j)\left(1-\tilde{p}_{i j}^{+}-\tilde{p}_{i j}^{-}\right), & i \neq j, i, j \in E, \\ \tilde{\Lambda}(i, i)-\tilde{\beta}_{i}^{+}-\tilde{\beta}_{i}^{-}, & i=j, i \in E, \\ \tilde{\beta}_{i}^{+} \tilde{\pi}_{i i}^{+}\left(k^{+}\right), & i \in E, j=\left(i i, k^{+}\right), \\ \tilde{\beta}_{i}^{-} \tilde{\pi}_{i i}^{-}\left(k^{-}\right), & i \in E, j=\left(i i, k^{-}\right), \\ \tilde{\Lambda}(i, k) \tilde{p}_{i k}^{+} \tilde{\pi}_{i k}^{+}\left(l^{+}\right), & i, k \in E, j=\left(i k, l^{+}\right), \\ \tilde{\Lambda}(i, k) \tilde{p}_{i k}^{-} \tilde{\pi}_{i k}^{-}\left(l^{-}\right), & i, k \in E, j=\left(i k, l^{-}\right), \\ \tilde{T}_{s, t}^{+}\left(r^{+}, l^{+}\right), & i=\left(s t, r^{+}\right), j=\left(s t, l^{+}\right), \\ \tilde{T}_{s, t}^{-}\left(r^{-}, l^{-}\right), & i=\left(s t, r^{-}\right), j=\left(s t, l^{-}\right), \\ \tilde{t}_{s, t}^{+}\left(l^{+}\right), & i=\left(s t, l^{+}\right), j=t, \\ \tilde{t}_{s, t}^{-}\left(l^{-}\right), & i=\left(s t, l^{-}\right), j=t .\end{cases}
$$

Let $\boldsymbol{F}(\alpha)=\boldsymbol{Q}+\operatorname{diag}\left(c_{1} \alpha+\frac{1}{2} \sigma_{1}^{2} \alpha^{2}, \ldots, c_{n} \alpha+\frac{1}{2} \sigma_{n}^{2} \alpha^{2}, \alpha, \ldots, \alpha,-\alpha, \ldots,-\alpha\right)$. Note that we enumerate first the states in $E$, then the states of the negative claims (positive jumps), and then the states corresponding to the positive claims (negative jumps). Given that $R(0)=R^{f}(0)=u$ and $J(0)=J^{f}(0)=i$, then, under $\mathbb{P}^{\Phi(\delta)}, \gamma_{i, j}^{+}(u)=\gamma_{i, j}^{+}(u, 0)$ is the probability that $R^{f}$ hits $b$ before ruin at state $j$ by diffusion, and $\gamma_{i,\left(k j, l^{+}\right)}^{+}(u)$ is the probability that the fluid process hits $b$ before ruin by a negative claim arriving with state transition from state $k$ to state $j$ at phase $l^{+}$. Similarly, $\gamma_{i, j}^{-}(u)=\gamma_{i, j}^{-}(u, 0)$ is the probability that the fluid process hits 0 before reaching $b$, and $\gamma_{i,\left(k j, l^{-}\right)}^{-}(u)$ is the probability that the process hits 0 before $b$ by a claim arriving with transition from state $k$ to $j$ at phase $l^{-}$. Note that the hitting probabilities of the fluid process are the same as for the original process (under $\mathbb{P}^{\Phi(\delta)}$ ). Assume that the equation $|\boldsymbol{F}(\alpha)|=0$ has $N=n_{p}+n_{n}+2 n_{\sigma}+\sum_{i=1}^{n} \sum_{k=1}^{n}\left(m_{i k}^{+}+m_{i k}^{-}\right)$roots, $\alpha_{1}, \ldots, \alpha_{N}$. Let $\boldsymbol{h}^{f}\left(\alpha_{i}\right)$ be the right eigenvector corresponding to the 0 eigenvalue of $\boldsymbol{F}\left(\alpha_{i}\right)$. Applying the multidimensional Wald martingale we obtain the following equations for $\gamma_{i, j}^{ \pm}(u, 0)$ and $\gamma_{i,(k j, l)}^{ \pm}(u)$ :

$$
\begin{aligned}
\exp \left(\alpha_{r} u\right) h_{i}^{f}\left(\alpha_{r}\right)= & \sum_{j \in E} \gamma_{i, j}^{+}(u, 0) \exp \left(\alpha_{r} b\right) h_{j}^{f}\left(\alpha_{r}\right) \\
& +\sum_{k \in E} \sum_{j \in E} \sum_{l^{+}=1}^{m_{k j}^{+}} \gamma_{i,\left(k j, l^{+}\right)}^{+}(u) \exp \left(\alpha_{r} b\right) h_{\left(k j, l^{+}\right)}^{f}\left(\alpha_{r}\right) \\
& +\sum_{j \in E} \gamma_{i, j}^{-}(u, 0) h_{j}^{f}\left(\alpha_{r}\right)+\sum_{k \in E} \sum_{j \in E} \sum_{l^{-}=1}^{m_{k j}^{-}} \gamma_{i,\left(k j, l^{-}\right)}^{-}(u) h_{j}^{f}\left(\alpha_{r}\right) .
\end{aligned}
$$


For $i, j \in E$, let the $\boldsymbol{\gamma}_{i, k j}^{ \pm}(u)$ be row vectors, where

$$
\begin{aligned}
& \gamma_{i, k j}^{+}(u)=\left(\gamma_{i,\left(k j, 1^{+}\right)}^{+}(u), \ldots, \gamma_{i,\left(k j, m_{k j}^{+}\right)}^{+}(u)\right), \\
& \gamma_{i, k j}^{-}(u)=\left(\gamma_{i,\left(k j, 1^{-}\right)}^{-}(u), \ldots, \gamma_{i,\left(k j, m_{k j}^{-}\right)}^{-}(u)\right) .
\end{aligned}
$$

Given that $R(0)=u$ and $J(0)=i$, then, under $\mathbb{P}^{\Phi(\delta)}$, the distribution functions of the overflow above $b$ when hitting the level $b$ before ruin, and the distribution function of the deficit at ruin when ruin occurs before hitting $b$, are phase type with the following density functions: for $z>0$,

$$
\gamma_{i, j}^{+}(u, z)=\sum_{k \in E} \boldsymbol{\gamma}_{i, k j}^{+}(u) \exp \left(\tilde{\boldsymbol{T}}_{k j}^{+} z\right) \tilde{\boldsymbol{t}}_{k j}^{+}, \quad \gamma_{i, j}^{-}(u, z)=\sum_{k \in E} \boldsymbol{\gamma}_{i, k j}^{-}(u) \exp \left(\tilde{\boldsymbol{T}}_{k j}^{-} z\right) \tilde{\boldsymbol{t}}_{k j}^{-} .
$$

By applying (5.1) we obtain

$$
\begin{aligned}
& v_{i, j}^{+}(u, z)=h_{i}(\Phi(\delta)) \gamma_{i, j}^{+}(u, z)\left(h_{j}^{(\Phi(\delta))}\right)^{-1} \mathrm{e}^{-\Phi(\delta)(b-u+z)}, \\
& v_{i, j}^{+}(u, 0)=h_{i}(\Phi(\delta)) \gamma_{i, j}^{+}(u, 0)\left(h_{j}(\Phi(\delta))\right)^{-1} \mathrm{e}^{-\Phi(\delta)(b-u)}, \\
& v_{i, j}^{-}(u, z)=h_{i}(\Phi(\delta)) \gamma_{i, j}^{-}(u, z)\left(h_{j}(\Phi(\delta))\right)^{-1} \mathrm{e}^{\Phi(\delta)(u+z)}, \\
& v_{i, j}^{-}(u, 0)=h_{i}(\Phi(\delta)) \gamma_{i, j}^{+}(u, 0)\left(h_{j}(\Phi(\delta))\right)^{-1} \mathrm{e}^{\Phi(\delta) u} .
\end{aligned}
$$

Finally, for $i, j \in E$, let

$$
\begin{aligned}
& v_{i, j}^{+}(u, \cdot)=\mathbb{E}_{i, u}\left[\mathrm{e}^{-\delta \tau_{b}^{+}}, \tau_{b}^{+}<\tau_{0}^{-}, J\left(\tau_{b}^{+}\right)=j\right]=v_{i, j}^{+}(u, 0)+\int_{0}^{\infty} v_{i, j}^{+}(u, z) \mathrm{d} z, \\
& v_{i, j}^{-}(u, \cdot)=\mathbb{E}_{i, u}\left[\mathrm{e}^{-\delta \tau_{0}^{-}}, \tau_{0}^{-}<\tau_{b}^{+}, J\left(\tau_{0}^{-}\right)=j\right]=v_{i, j}^{-}(u, 0)+\int_{0}^{\infty} v_{i, j}^{-}(u, z) \mathrm{d} z .
\end{aligned}
$$

\subsection{The expected discounted dividends}

Assume that $R_{b}(0)=u$ and $J(0)=i$. Let the $v_{1, i}(u, b)$ be the expected discounted dividends received when the dividend barrier is reached by a negative claim before ruin:

$$
v_{1, i}(u, b)=\sum_{j} \int_{0}^{\infty} z v_{i, j}^{+}(u, z) \mathrm{d} z .
$$

Thus, for $0<u<b$,

$$
V_{i}(u, b)=v_{1, i}(u, b)+\sum_{j \in E} v_{i, j}^{+}(u, \cdot) V_{j}(b, b) .
$$

Similarly to (4.5), let

$$
\begin{aligned}
A i c_{i, j}^{p} & =\int_{0}^{b} r_{i}(x)\left(1-G_{i j}^{+}(x)\right) \mathrm{d} x, \\
A_{i, k, j} & =\int_{0}^{b} r_{i}(x) v_{k, j}^{+}(b-x, \cdot) \mathrm{d} x, \quad i \neq k, \\
A c_{i, k, j}^{n} & =\int_{0}^{b} \int_{y=0}^{x} r_{i}(y) g_{i k}^{-}(x-y) \mathrm{d} y v_{k, j}^{+}(b-x, \cdot) \mathrm{d} x, \\
A c_{i, k, j}^{p} & =\int_{0}^{b} \int_{y=x}^{b} r_{i}(y) g_{i k}^{+}(y-x) \mathrm{d} y v_{k, j}^{+}(b-x, \cdot) \mathrm{d} x .
\end{aligned}
$$


The quantity $A i c_{i, j}^{p}$ is the expected discounted time to reach level $b$ when the modulated state is $j$ at time $\varepsilon_{i}$ due to a negative claim arrival. The quantities $A_{i, k, j}, A_{i, k, j}^{n}$, and $A_{i, k, j}^{p}$ are respectively the expected discounted time to reach $b$ before ruin at state $j$ (when at time $\varepsilon_{i}$, there was a state transition from $i$ to $k$ without a claim), a transition from $i$ to $k$ with a positive claim, and a transition with a negative claim.

Proposition 5.1. The expected discounted dividends $V_{i}(b, b), i=1, \ldots, n$, satisfy

$$
\begin{aligned}
V_{i}(b, b)= & w_{i}+\sum_{k \in E} D_{1}^{+}(i, k) \int_{y=0}^{b} r_{i}(y) \int_{z=0}^{\infty} z g_{i k}^{+}(y+z) \mathrm{d} z \mathrm{~d} y \\
& +\sum_{k \in E} D_{1}^{+}(i, k) \int_{x=0}^{b} \int_{y=0}^{x} r_{i}(b-y) g_{i k}^{+}(x-y) \mathrm{d} y v_{1, k}(x, b) \mathrm{d} x \\
& +\sum_{k \in E} D_{1}^{-}(i, k) \int_{x=0}^{b} \int_{y=x}^{b} r_{i}(b-y) g_{i k}^{-}(y-x) \mathrm{d} y v_{1, k}(x, b) \mathrm{d} x \\
& +\sum_{k \in E, k \neq i} D_{0}(i, k) \int_{x=0}^{b} r_{i}(b-x) v_{1, k}(x) \mathrm{d} x \\
& +\sum_{j} D_{1}^{+}(i, j) A i c_{i, j}^{p} V_{j}(b, b) \\
& +\sum_{j \in E} \sum_{k \in E} D_{1}^{+}(i, k) A c_{i, k, j}^{p} V_{j}(b, b) \\
& +\sum_{j \in E} \sum_{k \in E} D_{1}^{-}(i, k) A c_{i, k, j}^{n} V_{j}(b, b) \\
& +\sum_{j \in E} \sum_{k \in E, k \neq i} D_{0}(i, k) A_{i, k, j} V_{j}(b, b) .
\end{aligned}
$$

Proof. The term $w_{i}$ is the expected discounted dividend up to time $T_{b} \wedge \varepsilon_{i}$, given by (3.3) or (3.4). The second expression in (5.4) is the expected discounted dividend paid at $\mathcal{E}_{i}$ due to a negative claim. The terms in (5.5)-(5.7) are the expected discounted dividends paid, when the surplus reaches $b$ due to a positive jump (negative claim) before ruin, after state transition at $\varepsilon_{i}$ with or without a claim. Similarly, (5.8)-(5.11) describe the expected discounted dividends paid after $\mathcal{E}_{i}$, when the process reaches $b$ either at time $\mathcal{E}_{i}$ or after time $\mathcal{E}_{i}$, where at this time there was either a negative claim, a positive claim, or a state transition without a claim.

\subsection{The distribution of the dividends for $\delta=0$}

In this subsection we assume that $\delta=0$, and we will show that the amount of dividends until ruin has a phase-type distribution. In this case $\gamma_{i, j}^{+}(u)$ and $\gamma_{i,\left(k j, l^{+}\right)}^{+}(u)$ obtained in (5.3) are the probabilities (under the original measure) that the surplus in the fluid model and, hence, the original surplus process hit the level $b$ by diffusion when the modulating state is $j$ or by a $k j$ th negative claim at phase $l^{+}$.

Proposition 5.2. Assume that $R_{b}(0)=b$ and $J(0)=i$. Then the amount of dividends paid until $T_{b, i} \wedge \mathcal{E}_{i}$ is exponentially distributed with mean

$$
\frac{1}{\hat{\mu}_{i}}=\frac{W^{\left(\eta_{i}\right)}(b)}{W^{\left(\eta_{i}\right)^{\prime}}(b)},
$$

where $\eta_{i}=-D_{0}(i, i)+D_{1}^{+}(i, i)+D_{1}^{-}(i, i)$. 
Proof. Let $R_{b}(0)=b$ and $J(0)=i$. Let $D(t)$ be the amount of dividends paid up to time $t$, and let $D^{-1}(x)=\inf \{t: D(t)>x\} ; D(t)$ is the local time at 0 of the process $b-R_{b}$ and $D^{-1}$ is the inverse local time.

The process $D^{-1}$ has independent stationary increments, i.e. $D^{-1}(x+y)-D^{-1}(x)$ is independent of $D^{-1}(x)$ and distributed as $D^{-1}(y)$ (see Bertoin (1996, p. 114)). Applying the Markov property at $D^{-1}(x)$ and the memoryless property of the exponential distribution we obtain, similarly to Bertoin (1996, Exercise IV.1, p. 123, or Exercise V.3, p. 144),

$$
\begin{aligned}
\mathbb{P}(D & \left.\left(T_{b, i} \wedge \mathcal{E}_{i}\right)>x+y\right) \\
& =\mathbb{P}\left(T_{b, i} \wedge \mathcal{E}_{i}>D^{-1}(x+y)\right) \\
& =\mathbb{P}\left(T_{b, i}>D^{-1}(x+y)\right) \mathbb{P}\left(\mathcal{E}_{i}>D^{-1}(x+y)\right) \\
& =\mathbb{P}\left(T_{b, i}>D^{-1}(x)\right) \mathbb{P}\left(T_{b, i}>D^{-1}(y)\right) \mathbb{P}\left(\mathcal{E}_{i}>D^{-1}(x)\right) \mathbb{P}\left(\mathcal{E}_{i}>D^{-1}(y)\right) \\
& =\mathbb{P}\left(T_{b, i} \wedge \mathcal{E}_{i}>D^{-1}(x)\right) \mathbb{P}\left(T_{b, i} \wedge \mathcal{E}_{i}>D^{-1}(y)\right) \\
& =\mathbb{P}\left(D\left(T_{b, i} \wedge \mathcal{E}_{i}\right)>x\right) \mathbb{P}\left(D\left(T_{b, i} \wedge \mathcal{E}_{i}\right)>y\right)
\end{aligned}
$$

The expected dividend up to time $T_{b, i} \wedge \mathcal{E}_{i}$ is given by (5.12).

Let $i \in E$ and $\tilde{j} \in E \cup E_{+}, R_{b}(0)=b$ and $J(0)=i \in E$. Let $\Pi(i, \tilde{j})$ be the probability that the process reaches $b$ after $\mathcal{E}_{i}$ and before ruin at state $\tilde{j}$. We have

$$
\begin{aligned}
\Pi(i, \tilde{j})= & \sum_{k \in E} D_{1}^{+}(i, k) \int_{x=0}^{b} \int_{y=b-x}^{b} r_{i}(b-y) g_{i k}^{+}(x-y) \mathrm{d} y \gamma_{k, \tilde{j}}^{+}(x) \mathrm{d} x \\
& +\sum_{k \in E} D_{1}^{-}(i, k) \int_{x=0}^{b} \int_{y=0}^{x} r_{i}(b-y) g_{i k}^{-}(y-x) \mathrm{d} y \gamma_{k, \tilde{j}}^{+}(x) \mathrm{d} x \\
& +\sum_{j \in E} \sum_{k \in E, k \neq i} D_{0}(i, k) \int_{x=0}^{b} r_{i}(b-x) \gamma_{k, \tilde{j}}^{+}(x) \mathrm{d} x .
\end{aligned}
$$

Given that $R_{b}(0)=b$ and $J(0)=i \in E$, the amount of dividends paid until time $\varepsilon_{i} \wedge T_{b, i}$ is exponentially distributed with parameter $\hat{\mu}_{i}$. The process returns to $b$ at state $i$ with probability $\Pi(i, i)$. Thus, the amount of dividend earned until the first time the process reaches $b$ at state $\tilde{j} \neq i$ or until ruin is a geometric compound sum of i.i.d. exponentially distributed random variables and, thus, exponentially distributed with parameter $\mu_{i}=(1-\Pi(i, i)) \hat{\mu}_{i}$. Upon exiting state $i \in E$ the next state is $\tilde{j} \neq i$, where $\tilde{j}$ is either $j \in E$ or $\tilde{j}=\left(l j, o^{+}\right)$, $l, j \in E$, with probability $\Pi(i, \tilde{j}) /(1-\Pi(i, i))$. Thus, when in phase $i \in E$, the amount of dividend is exponentially distributed with parameter $\mu_{i}$, and, when in phase $\left(l j, o^{+}\right)$, it is exponentially distributed with rate $-T_{l j}^{+}\left(o^{+}, o_{\tilde{j}}^{+}\right)$. When in state (phase) $i \in E$, the transition rate to state $\tilde{j} \neq i$ is $\Upsilon(i, \tilde{j})=\mu_{i} \Pi(i, \tilde{j})$. When in state $\left(l j, o^{+}\right)$, the transition rate to state $\left(l j, \tilde{o}^{+}\right)$is $\Upsilon\left(\left(l j, o^{+}\right),\left(l j, \tilde{o}^{+}\right)\right)=T_{l j}^{+}\left(o^{+}, \tilde{o}^{+}\right)$, and the transition rate to state $j$ is $\Upsilon\left(\left(l j, o^{+}\right), j\right)=t_{l j}^{+}\left(o^{+}\right)$. Thus, the transition rate matrix among the transient states is $\Upsilon$. Assume that $R(0)=u$ and that $\mathbb{P}(J(0)=i)=\alpha_{i}$. Then the amount of dividends is phase type

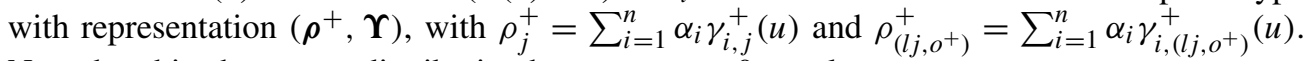
Note that this phase-type distribution has an atom at 0 equal to

$$
1-\sum_{i} \alpha_{i} \sum_{\tilde{j}} \gamma_{i, \tilde{j}}^{+}(u)=\sum_{i} \sum_{\tilde{j}} \alpha_{i} \gamma_{i, \tilde{j}}^{-}(u),
$$


which is the probability of ruin occurring before reaching $b$. We conclude with the following proposition.

Proposition 5.3. The amount of dividends paid until ruin is phase type. When $R(0)=u$ and $\mathbb{P}(J(0)=i)=\alpha_{i}, i \in E$, it is phase type with representation $\left(\rho^{+}, \Upsilon\right)$, and the atom at 0 is given by (5.13).

\subsection{The expected discounted penalty function}

We consider the expected discounted penalty function for the Markov additive risk process with two-sided jumps. Similarly to (4.7), we express $\omega_{i}(u, b), u<b$, as a function of $\omega_{j}(b, b)$, $j \in E$.

Similarly to Proposition 4.1 , we derive $\omega_{i}(b, b)$ for the case with positive and negative claims.

Proposition 5.4. The penalty functions $\omega_{i}(b, b), i=1, \ldots, n$, satisfy

$$
\begin{aligned}
\omega_{i}(b, b)= & w_{1, i}(b)+\sum_{k \in E} D_{1}^{-}(i, k) \int_{x=0}^{b} r_{i}(x) \int_{0}^{\infty} f(z) g_{i k}^{-}(b-x+z) \mathrm{d} z \mathrm{~d} x \\
& +\sum_{k \in E} D_{1}^{-}(i, k) \int_{x=0}^{b} \int_{y=0}^{b-x} r_{i}(y) g_{i k}^{-}(b-x-y) \mathrm{d} y \omega_{0, k}(x, b) \mathrm{d} x \\
& +\sum_{j \in E} \sum_{k \in E} D_{1}^{-}(i, k) A c_{i, k, j}^{n} \omega_{j}(b, b) \\
& +\sum_{k \in E} D_{1}^{+}(i, k) \int_{x=0}^{b} \int_{y=b-x}^{b} r_{i}(y) g_{i k}^{+}(x+y-b) \mathrm{d} y \omega_{0, k}(x, b) \mathrm{d} x \\
& \left.+\sum_{k \in E} D_{1}^{+}(i, k) A i c_{i, k}^{p}+\sum_{j \in E} \sum_{k \in E} D_{1}^{+}(i, k) A c_{i, k, j}^{p}\right) \omega_{j}(b, b) \\
& +\sum_{k \in E, k \neq i} D_{0}(i, k) \int_{0}^{b} r_{i}(x) \omega_{0, k}(b-x, b) \mathrm{d} x \\
& +\sum_{j \in E} \sum_{k \in E, k \neq i} D_{0}(i, k) A_{i, k, j} \omega_{j}(b, b) .
\end{aligned}
$$

The proof is similar to that of Proposition 4.1 and is therefore omitted.

\subsection{The distribution of the deficit at ruin for $\delta=0$}

It is clear that the deficit at ruin is phase type with representation $\left(\varrho, \boldsymbol{T}^{-}\right)$, where $\boldsymbol{T}^{-}$ is a block matrix with matrices $\boldsymbol{T}_{i j}$ on the diagonal, i.e. $\boldsymbol{T}^{-}=\operatorname{diag}\left(\boldsymbol{T}_{i j}^{-}\right)$. Let $\gamma_{i, p}^{+}(u, \cdot)=$ $\gamma_{i, p}^{+}(u, 0)+\sum_{k \in E} \sum_{l^{+}=1}^{m_{k}^{+}} \gamma_{\left.\left(i, k p, l^{+}\right)\right)}^{+}(u)$. Denote by $a^{-}\left(i,\left(k j, l^{-}\right)\right)(u)$ and $a^{-}(i, 0)(u)$ the probabilities that ruin occurs by the $k j$ th claim at phase $l^{-}$or by diffusion, given that $R(0)=u$ and $J(0)=i$ :

$$
\begin{aligned}
& a^{-}\left(i,\left(k j, l^{-}\right)\right)(u)=\gamma_{i,(k j, l)}^{-}(u)+\sum_{p \in E} \gamma_{i, p}^{+}(u, \cdot) a^{-}(p,(k j, l))(b), \\
& a^{-}(i, 0)(u)=\sum_{j \in E} \gamma_{i, j}^{-}(u)+\sum_{p \in E} \gamma_{i, p}^{+}(u, \cdot) a^{-}(p, 0)(b) .
\end{aligned}
$$


Let $\mathbf{1}_{i}$ be a column vector with a 1 in the $i$ th entry and 0 s elsewhere. Next we find the probabilities $a^{-}\left(i,\left(k j, l^{-}\right)\right)(b)$. We apply the same notation as in (4.5). We have

$$
\begin{aligned}
a^{-}\left(i,\left(p q, l^{-}\right)\right)(b)= & D_{1}^{-}(i, q) \int_{x=0}^{b} r_{i}(b-x) \boldsymbol{\pi}_{i q}^{-} \exp \left(\boldsymbol{T}_{i q}^{-} x\right) \mathbf{1}_{l^{-}} \mathrm{d} x I(p=i) \\
& +\sum_{k \in E} D_{1}^{-}(i, k) \int_{x=0}^{b} \int_{y=0}^{b-x} r_{i}(y) g_{i k}^{-}(b-x-y) \mathrm{d} y \gamma_{k,\left(p q, l^{-}\right)}^{-}(x) \mathrm{d} x \\
& +\sum_{k \in E} \sum_{j \in E} D_{1}^{-}(i, k) A c_{i, k, j}^{n} a^{-}\left(j,\left(p q, l^{-}\right)\right)(b) \\
& +\sum_{k \in E} D_{1}^{+}(i, k) \int_{x=0}^{b} \int_{y=b-x}^{b} r_{i}(y) g_{i k}^{+}(x+y-b) \mathrm{d} y \gamma_{k,\left(p q, l^{-}\right)}^{-}(x) \mathrm{d} x \\
& +\sum_{k \in E} D_{1}^{+}(i, k) \int_{0}^{b} r_{i}(y)\left(1-G_{i k}^{+}(y)\right) \mathrm{d} y a^{-}\left(k,\left(p q, l^{-}\right)\right)(b) \\
& +\sum_{j \in E} \sum_{k \in E} D_{1}^{+}(i, k) A c_{i, k, j}^{p} a^{-}\left(j,\left(p q, l^{-}\right)\right)(b) \\
& +\sum_{k \in E, k \neq i} D_{0}(i, k) \int_{0}^{b} r_{i}(x) \gamma_{k,\left(p q, l^{-}\right)}^{-}(b-x) \mathrm{d} x \\
& \left.+\sum_{j \in E} \sum_{k \in E, k \neq i} D_{0}(i, k) A_{i, k, j} a^{-}\left(j,\left(p q, l^{-}\right)\right)(b) .18\right)
\end{aligned}
$$

The term in (5.14) is the probability that at time $\mathcal{E}_{i}$ ruin occurs due to an $i q$ th positive claim at phase $l^{-}$. The terms in (5.15), (5.17), and (5.20) are the probabilities that ruin occurs before reaching $b$ by a $p q$ th claim hitting 0 at phase $l^{-}$. The terms in (5.16), (5.18), (5.19), and (5.21) are the probabilities that ruin occurs at state $\left(p q, l^{-}\right)$when the process reaches $b$ before ruin, after $\varepsilon_{i}$. Similarly, the $a^{-}(i, 0)(b), i=1, \ldots, n$, satisfy

$$
\begin{aligned}
a^{-}(i, 0)(b)= & \mathcal{L}_{T_{b}}\left(\eta_{i}\right) \\
& +\sum_{k \in E} D_{1}^{-}(i, k) \int_{x=0}^{b} \int_{y=0}^{b-x} r_{i}(y) g_{i k}^{-}(b-x-y) \mathrm{d} y \sum_{j \in E} \gamma_{k, j}^{-}(x) \mathrm{d} x \\
& +\sum_{k \in E} \sum_{j \in E} D_{1}^{-}(i, k) A c_{i, k, j}^{n} a^{-}(j, 0)(b) \\
& +\sum_{k \in E} D_{1}^{+}(i, k) \int_{x=0}^{b} \int_{y=b-x}^{b} r_{i}(y) g_{i k}^{+}(x+y-b) \mathrm{d} y \sum_{j \in E} \gamma_{k, j}^{-}(x) \mathrm{d} x \\
& +\sum_{k \in E} D_{1}^{+}(i, k) \int_{0}^{b} r_{i}(y)\left(1-G_{i k}^{+}(y)\right) \mathrm{d} y a^{-}(k, 0)(b) \\
& +\sum_{j \in E} \sum_{k \in E} D_{1}^{+}(i, k) A c_{i, k, j}^{p} a^{-}(j, 0)(b)
\end{aligned}
$$




$$
\begin{aligned}
& +\sum_{k \in E, k \neq i} D_{0}(i, k) \int_{0}^{b} r_{i}(x) \sum_{j \in E} \gamma_{k, j}^{-}(b-x) \mathrm{d} x \\
& +\sum_{j \in E} \sum_{k \in E, k \neq i} D_{0}(i, k) A_{i, k, j} a^{-}(j, 0)(b) .
\end{aligned}
$$

The term $\mathcal{L}_{T_{b, i}}\left(\eta_{i}\right)$ is the probability of ruin before $\varepsilon_{i}$ due to diffusion. Note that, when $\sigma_{i}=0$, this probability is 0 . The other terms have a similar interpretation as before.

If $\mathbb{P}(J(0)=i)=\alpha_{i}$ and $R(0)=u$, then the deficit at ruin is phase type with representation $\left(\varrho, \boldsymbol{T}^{-}\right)$, where

$$
\varrho_{(k j, l)}=\sum_{i \in E} \alpha_{i} a^{-}(i,(k j, l))(u) .
$$

The atom at 0 is $\sum_{i \in E} \alpha_{i} a^{-}(i, 0)(u)$.

\section{Example}

In this section we present a methodological example. We consider a two-state Markov additive risk process with $\delta=0.04, \beta_{1}=0.03, \beta_{2}=0.14, \sigma_{2}=0.1, \sigma_{1}=0, c_{1}=c_{2}=3$,

$$
\begin{gathered}
\boldsymbol{\Lambda}=\left(\begin{array}{cc}
-0.015 & 0.015 \\
0.06 & -0.06
\end{array}\right), \quad \boldsymbol{D}_{1}=\left(\begin{array}{cc}
0.03 & 0 \\
0 & 0.14
\end{array}\right), \\
\text { and } \boldsymbol{D}_{0}=\left(\begin{array}{cc}
-0.045 & 0.015 \\
0.06 & -0.2
\end{array}\right) .
\end{gathered}
$$

When in state 1 , negative claims arrive according to a Poisson process at rate $\beta_{1}=0.03$ and the claim amounts are exponentially distributed with $\mu_{1}=0.5$. When in state 2 , positive claims arrive according to a Poisson process at rate $\beta_{2}=0.14$ and the positive claim amounts are exponentially distributed with $\mu_{2}=0.05$. Thus, when in state 1 , the process increases linearly and has only positive jumps, while when in state 2 , the process evolves as a Brownian motion with drift 3 between negative jumps. The matrix $\boldsymbol{K}(\alpha)$ is

$$
\begin{aligned}
\boldsymbol{K}(\alpha) & =\boldsymbol{\Lambda}+\left(\begin{array}{cc}
3 \alpha+0.03\left(\frac{0.5}{0.5-\alpha}-1\right) & 0 \\
0 & 3 \alpha+0.005 \alpha^{2}+0.14\left(\frac{0.05}{0.05+\alpha}-1\right)
\end{array}\right) \\
& =\left(\begin{array}{cc}
-0.045+3 \alpha+\frac{0.015}{0.5-\alpha} & 0.015 \\
0.06 & -0.2+3 \alpha+0.005 \alpha^{2}+\frac{0.007}{0.05+\alpha}
\end{array}\right) .
\end{aligned}
$$

Next we find the value $\Phi(\delta)$, which is the root of the equation $\operatorname{det}(\boldsymbol{K}(\alpha)-\delta \boldsymbol{I})=0$ for which $\delta$ is an eigenvalue with maximal real part. Such a root is $\Phi(\delta)=0.014616$. The corresponding eigenvector is

$$
\left(\begin{array}{l}
0.825686 \\
0.564131
\end{array}\right) \text {. }
$$

Next we define the MAP parameters under $\mathbb{P}^{\Phi(\delta)}$ :

$$
\begin{gathered}
\tilde{c}_{1}=c_{1}=3, \quad \tilde{c}_{2}=c_{2}+\sigma_{2}^{2} \Phi(\delta)=3.00014616, \\
\tilde{\boldsymbol{\Lambda}}=\boldsymbol{\Delta}_{\boldsymbol{h}(\Phi(\delta))}^{-1} \boldsymbol{K}(\Phi(\delta)) \boldsymbol{\Delta}_{\boldsymbol{h}(\Phi(\delta))}-\delta \boldsymbol{I}=\left(\begin{array}{cc}
-0.0102 & 0.0102 \\
0.0878 & -0.0878
\end{array}\right),
\end{gathered}
$$




$$
\tilde{\beta}_{1}=\beta_{1} \frac{\mu_{1}}{\mu_{1}-\Phi(\delta)}=0.030903, \quad \text { and } \quad \tilde{\beta}_{2}=\beta_{2} \frac{\mu_{2}}{\mu_{2}+\Phi(\delta)}=0.108332
$$

The negative claim is exponentially distributed with rate $\tilde{\mu}_{1}=\mu_{1}-\Phi(\delta)=0.485384$, and the positive claim is exponentially distributed with rate $\tilde{\mu}_{2}=\mu_{2}+\Phi(\delta)=0.064616$. The transition rate matrix of the fluid model is

$$
\begin{aligned}
\boldsymbol{Q} & =\left(\begin{array}{cccc}
\tilde{\Lambda}(1,1)-\tilde{\beta}_{1} & \tilde{\Lambda}(1,2) & \tilde{\beta}_{1} & 0 \\
\tilde{\Lambda}(2,1) & \tilde{\Lambda}(2,2)-\tilde{\beta}_{2} & 0 & \tilde{\beta}_{2} \\
\tilde{\mu}_{1} & 0 & -\tilde{\mu}_{1} & 0 \\
0 & \tilde{\mu}_{2} & 0 & -\tilde{\mu}_{2}
\end{array}\right) \\
& =\left(\begin{array}{cccc}
-0.0412 & 0.0102 & 0.0309 & 0 \\
0.0878 & -0.1962 & 0 & 0.1083 \\
0.4854 & 0 & -0.4854 & 0 \\
0 & 0.0646 & 0 & -0.0646
\end{array}\right) .
\end{aligned}
$$

The matrix $\boldsymbol{F}$ for our example is

$$
\begin{aligned}
\boldsymbol{F}(\alpha) & =\boldsymbol{Q}+\left(\begin{array}{cccc}
\tilde{c}_{1} \alpha & 0 & 0 & 0 \\
0 & \tilde{c}_{2} \alpha+\frac{1}{2} \sigma_{2}^{2} & 0 & 0 \\
0 & 0 & \alpha & 0 \\
0 & 0 & 0 & -\alpha
\end{array}\right) \\
& =\left(\begin{array}{cccc}
\alpha-0.04112 & 0.0102 & 0.0309 & 0 \\
0.0878 & 3.0001 \alpha+0.005 \alpha^{2}-0.196151 & 0 & 0.108332 \\
0.4854 & 0 & \alpha-0.4854 & 0 \\
0 & 0.0646 & 0 & -\alpha-0.0646
\end{array}\right) .
\end{aligned}
$$

The equation $|\boldsymbol{F}(\alpha)|=0$ has the five solutions

$$
\begin{aligned}
& \alpha_{1}=0, \\
& \alpha_{2}=0.046665, \\
& \alpha_{3}=0.495761, \\
& \alpha_{4}=-0.042564, \\
& \alpha_{5}=-600.094609,
\end{aligned}
$$

whose corresponding eigenvectors are

$$
\begin{aligned}
& \boldsymbol{h}\left(\alpha_{1}\right)=(1,1,1,1,1)^{\top} \\
& \boldsymbol{h}\left(\alpha_{2}\right)=(-0.066294,0.860548,-0.073345,0.499683)^{\top}, \\
& \boldsymbol{h}\left(\alpha_{3}\right)=(-0.021375,0.001438,0.999770,0.000166)^{\top}, \\
& \boldsymbol{h}\left(\alpha_{4}\right)=(-0.023559,-0.322821,-0.021659,-0.945919)^{\top}, \\
& \boldsymbol{h}\left(\alpha_{5}\right)=\left(0.569253 \times 10^{-5}, 1.000000,0.460066 \times 10^{-8},-0.000108\right)^{\top},
\end{aligned}
$$

where ${ }^{~} T$, denotes the transpose. We can now obtain the 10 equations for $\gamma_{i, j}^{ \pm}(u)$ and $\gamma_{i, k j, 1)}^{ \pm}(u)$.

Note that in this example ruin cannot occur at state 1. 


\section{TABle 1.}

\begin{tabular}{rll}
\hline$u$ & $V_{1}(u, 50)$ & $V_{2}(u, 50)$ \\
\hline 5 & 35.440 & 18.136 \\
10 & 38.270 & 21.003 \\
25 & 47.9975 & 30.104 \\
40 & 59.854 & 40.914 \\
50 & 69.252 & 49.926 \\
\hline
\end{tabular}

The scale functions are

$$
\begin{aligned}
W^{\left(\eta_{2}+\delta\right)}(x) & =0.3332444800 \mathrm{e}^{0.07998930000 x}-0.3332444800 \mathrm{e}^{-600.0799893 x}, \\
W^{\left(\eta_{1}+\delta\right)}(x) & =\frac{1}{3} \mathrm{e}^{0.02833333333 x}, \\
r_{2}(x) & =2500.334419 \mathrm{e}^{-600.0799893 x}, \\
r_{1}(0) & =\frac{1}{\eta_{1}+\delta}=11.76470588,
\end{aligned}
$$

and $r_{1}(x)=0$ for $x>0$, and

$$
w_{1}=\frac{c_{1}}{\delta+\eta_{1}}=35.294118, \quad w_{2}=12.50167210 .
$$

Ruin cannot occur up to time $\mathcal{E}_{1}$ ( ince $\sigma_{1}=0$ ); thus, $\mathcal{L}_{T_{b, 1}}\left(\eta_{1}+\delta\right)=0$. Upon calculating all the coefficients we obtain the following equations for $V_{i}(b, b)$ :

$$
\begin{aligned}
& V_{1}(b, b)=36.00000000+0.3529411764 V_{1}(b, b)+0.1764705882 V_{2}(b, b), \\
& V_{2}(b, b)=12.55766086+0.3446610600 V_{1}(b, b)+0.2703915267 V_{2}(b, b) .
\end{aligned}
$$

Solving these equations we obtain

$$
V_{1}(b, b)=69.252, \quad V_{2}(b, b)=49.926 .
$$

The discounted dividends paid for different initial conditions are given in Table 1.

The transition intensity matrix and the positive claim amount distribution is as in Cheung and Laundriault (2009). We omitted claims with state transitions and added negative claims. Thus, the discounted amount of dividends paid is greater than in Cheung and Laudriault's example.

\section{A barrier strategy dependent on the environmental state $J$}

Following Cheung and Landriault (2009), who were motivated by Zhu and Yang (2008), we assume that the dividend barrier is state dependent. The barrier strategy, effective when $J$ is in state $i$, is $b_{i}, i \in E$. For the application of this strategy, see, e.g. Zhu and Yang (2008).

The method developed in this paper is based on the semiregenerative property of the process, and the change of measure. These tools cannot be implemented directly for a state-dependent barrier strategy with an arbitrary number of states. When there are only two states, 1 and 2, we can still obtain solutions for the case where there are only positive claims or only negative claims, but not both. Without loss of generality, we assume that $0<b_{1}<b_{2}$. In the case of two barriers we denote the process by $R_{\boldsymbol{b}}$, with $\boldsymbol{b}=\left(b_{1}, b_{2}\right)$. 


\subsection{Positive claims}

In this subsection we consider a risk process with negative jumps only.

7.1.1. Expected discounted dividends. Let $V_{i}(u, \boldsymbol{b})$ be the expected discounted dividends paid when $J(0)=i$ and $R_{\boldsymbol{b}}(0)=u$. Let $\beta_{2}=D_{1}(2,2)$. On $J=2$ the process behaves as a Lévy process, $\tilde{X}_{2}$, with exponent

$$
\varphi_{2}(\alpha)=c_{2}+\frac{1}{2} \sigma_{2}^{2} \alpha^{2}+\beta_{2}\left(\boldsymbol{\pi}_{22}\left(\alpha \boldsymbol{I}-\boldsymbol{T}_{22}\right)^{-1} \boldsymbol{t}_{22}-1\right) .
$$

For $J(0)=2$, let $\tilde{\mathcal{E}}_{2}$ be the transition time of the CTMC $J$ to state 1 . Clearly, $\tilde{\mathcal{E}}_{2}$ is exponentially distributed with parameter $\lambda_{21}=D_{1}(2,1)+D_{0}(2,1)$, independent of $\tilde{X}_{2}$. Let $W_{2}^{(q)}$ be the scale function for $\tilde{X}_{2}$. The forms of $W_{2}^{(q)}(x)$, its derivative $W_{2}^{(q)^{\prime}}(x)$, and the adjoint scale function $Z_{2}^{(q)}(x)$ are given explicitly as polynomials in $x$, with coefficients that are functions of the roots with negative real part of the equation $\varphi_{2}(\alpha)=q$; see Proposition 2.1 of Egami and Yamazaki (2012). Let

$$
\begin{aligned}
& \tilde{\tau}_{b}^{+}=\inf \left\{t: \tilde{X}_{2}(t) \geq b\right\}, \\
& \tilde{\tau}_{a}^{-}=\inf \left\{t: \tilde{X}_{2}(t) \leq a\right\} .
\end{aligned}
$$

Define the following Laplace transforms for the exit times: for $a<u<b$,

$$
\begin{aligned}
& \tilde{v}_{22}^{(a, b)+}(u, b)=\mathbb{E}\left[\mathrm{e}^{-\delta \tilde{\tau}_{b}^{+}} I\left(\tilde{\tau}_{a}^{-}>\tilde{\tau}_{b}^{+}, \tilde{\tau}_{b}^{+}<\tilde{\mathcal{E}}_{2}\right) \mid \tilde{X}_{2}(0)=u, J(0)=2\right], \\
& \tilde{v}_{22}^{(a, b)-}(u, a)=\mathbb{E}\left[\mathrm{e}^{-\delta \tilde{\tau}_{a}^{-}} I\left(\tilde{\tau}_{a}^{-}<\tilde{\tau}_{b}^{+}, \tilde{\tau}_{a}^{-}<\tilde{\mathcal{E}}_{2}\right) \mid \tilde{X}_{2}(0)=u, J(0)=2\right] .
\end{aligned}
$$

Since $\tilde{\mathcal{E}}_{2}$ and $\tilde{X}_{2}$ are independent,

$$
\begin{aligned}
& \tilde{v}_{22}^{(a, b)+}(u, b)=\mathbb{E}\left[\mathrm{e}^{-\left(\delta+\lambda_{21}\right) \tilde{\tau}_{b}^{+}} I\left(\tilde{\tau}_{a}^{-}>\tilde{\tau}_{b}^{+}\right) \mid X_{2}(0)=u\right], \\
& \tilde{v}_{22}^{(a, b)-}(u, a)=\mathbb{E}\left[\mathrm{e}^{-\left(\delta+\lambda_{21}\right) \tilde{\tau}_{a}^{-}} I\left(\tilde{\tau}_{a}^{-}<\tilde{\tau}_{b}^{+}\right) \mid X_{2}(0)=u\right] .
\end{aligned}
$$

By Proposition 2.1,

$$
\begin{aligned}
& \tilde{v}_{22}^{(a, b)+}(u, b)=\frac{W_{2}^{\left(\delta+\lambda_{21}\right)}(u-a)}{W_{2}^{\left(\delta+\lambda_{21}\right)}(b-a)}, \\
& \tilde{v}_{22}^{(a, b)-}(u, a)=Z_{2}^{\left(\delta+\lambda_{21}\right)}(u-a)-W_{2}^{\left(\delta+\lambda_{21}\right)}(u-a) \frac{Z_{2}^{\left(\delta+\lambda_{21}\right)}(u-a)}{W_{2}^{\left(\delta+\lambda_{21}\right)}(b-a)} .
\end{aligned}
$$

For $a<x, y<b$, let

$$
\lambda_{21} \tilde{u}_{21}^{(a, b)}(x, y) \mathrm{d} y=\mathbb{E}\left[\mathrm{e}^{-\delta \tilde{\varepsilon}_{2}} I\left(\tilde{\mathcal{E}}_{2}<\tilde{\tau}_{a}^{-} \wedge \tilde{\tau}_{b}^{+}, \tilde{X}_{2}\left(\tilde{\mathcal{E}}_{2}\right) \in \mathrm{d} y\right) \mid \tilde{X}_{2}(0)=x\right] .
$$

To obtain $\tilde{u}_{21}^{(a, b)}(x, y)$, we apply Theorem 8.7 of Kyprianou (2006):

$$
\tilde{u}_{21}^{(a, b)}(x, y)=\frac{W_{2}^{\left(\delta+\lambda_{21}\right)}(x-a) W_{2}^{\left(\delta+\lambda_{21}\right)}(b-y)}{W_{2}^{\left(\delta+\lambda_{21}\right)}(b-a)}-W_{2}^{\left(\delta+\lambda_{21}\right)}(x-y) .
$$

Throughout this section, we denote by $w_{i}^{(a)}, i=1,2, a>0$, the expected discounted dividends until $\mathcal{E}_{i} \wedge T_{a}$, as given by (3.3) or (3.4) with $a$ replacing $b$. Similarly, $r_{i}^{(a)}$ is as defined above Proposition 3.1, where $b$ is as defined in (2.3)-(2.5). Let $\tilde{M}_{2}(t)=\sup _{0 \leq s \leq t}\left(\tilde{X}_{2}(s)\right)$, and 
let $\tilde{Y}_{2}(t)=\tilde{M}_{2}(t)-\tilde{X}_{2}(t)$ be the reflected process at the maximum. Let $\tilde{T}_{b_{2}}$ be the time until the reflected process $\tilde{Y}_{2}$ hits $b_{2}$. On $J=2$ the process $b_{2}-R_{\boldsymbol{b}}$ behaves as $\tilde{Y}_{2}$. Let $\tilde{w}_{2}$ be the expected discounted dividends paid until $\tilde{T}_{b_{2}} \wedge \tilde{\mathcal{E}}_{2}$. By Theorem 2.1 of Avram et al. (2004),

$$
\tilde{w}_{2}=\mathbb{E}\left[\int_{0}^{\tilde{T}_{b_{2}} \wedge \tilde{\varepsilon}_{2}} \mathrm{e}^{-\delta s} \mathrm{~d} \tilde{M}_{2}(s)\right]=\frac{W_{2}^{\left(\delta+\lambda_{21}\right)}\left(b_{2}\right)}{W_{2}^{\left(\delta+\lambda_{21}\right)^{\prime}}\left(b_{2}\right)} .
$$

For $b>0$, let

$$
\lambda_{21} \tilde{r}_{2}^{(b)}(y) \mathrm{d} y=\mathbb{P}\left(b-R_{\boldsymbol{b}}\left(\tilde{\mathcal{E}}_{2}\right) \in \mathrm{d} y, \tilde{\mathcal{E}}_{2}<\tilde{T}_{b_{2}} \mid R_{\boldsymbol{b}}(0)=b, J(0)=2\right) .
$$

By Theorem 1(ii) of Pistorius (2004),

$$
\tilde{r}_{2}^{(b)}(y)=\left(W_{2}^{\left(\delta+\lambda_{21}\right)}(b) \frac{W_{2}^{\left(\delta+\lambda_{21}\right)^{\prime}}(y)}{W_{2}^{\left(\delta+\lambda_{21}\right)^{\prime}}(b)}-W_{2}^{\left(\delta+\lambda_{21}\right)}(y)\right) .
$$

Let $v_{i, j}^{+}\left(u, b_{1}\right)$ be the same as $v_{i, j}^{+}(u)$ defined in (4.1), where $b$ is replaced by $b_{1}$. For $b_{1} \leq u<b_{2}$ and $J(0)=2$, we define

$$
\begin{aligned}
v_{11}(u) & =\int_{x=b_{1}}^{b_{2}} \int_{y=b_{1}}^{x} \tilde{u}_{21}^{\left(0, b_{2}\right)}(u, x) g_{21}(x-y)\left(y-b_{1}\right) \mathrm{d} y \mathrm{~d} x, \\
v_{10}(u) & =\int_{x=b_{1}}^{b_{2}} \tilde{u}_{21}^{\left(0, b_{2}\right)}(u, x)\left(x-b_{1}\right) \mathrm{d} x, \\
\tilde{A}_{1, d, i}(u) & =\int_{x=0}^{b_{1}} \int_{y=x}^{b_{2}} \tilde{u}_{21}^{\left(0, b_{2}\right)}(u, y) g_{21}(y-x) v_{1, i}^{+}\left(x, b_{1}\right) \mathrm{d} y \mathrm{~d} x, \quad i=1,2, \\
\tilde{A}_{1, u, 1}(u) & =\int_{x=b_{1}}^{b_{2}} \int_{y=b_{1}}^{x} \tilde{u}_{21}^{\left(0, b_{2}\right)}(u, x) g_{21}(x-y) \mathrm{d} y \mathrm{~d} x, \\
\tilde{A}_{0, d, i}(u) & =\int_{0}^{b_{1}} \tilde{u}_{21}^{\left(0, b_{2}\right)}(u, x) v_{1, i}^{+}\left(x, b_{1}\right) \mathrm{d} x, \quad i=1,2, \\
\tilde{A}_{0, u, 1}(u) & =\int_{x=b_{1}}^{b_{2}} \tilde{u}_{21}^{\left(0, b_{2}\right)}(u, x) \mathrm{d} x .
\end{aligned}
$$

Given that $R_{\boldsymbol{b}}(0)=u$ and $J(0)=2, v_{11}(u)$ and $v_{10}(u)$ are the expected discounted dividends paid at time $\tilde{\mathcal{E}}_{2}$, when a transition to state 1 , with or, respectively, without a claim occurs before ruin, and the surplus after that transition is above $b_{1}$ and then drops to $b_{1}$. The quantities $\tilde{A}_{1, d, i}(u)$ and $\tilde{A}_{0, d, i}(u)$ are the expected discounted times until the reserve reaches $b_{1}$ from below at state $i, i=1,2$, where, after the first transition to state 1 that occurs with or without a claim arrival, the reserve is below $b_{1}$. Similarly, $\tilde{A}_{1, u, 1}(u)$ and $\tilde{A}_{0, u, 1}(u)$ are the expected discounted times until the reserve reaches $b_{1}$ from above at state 1 , where, after the transition, the reserve is between $b_{1}$ and $b_{2}$.

Similarly, to obtain $V_{2}\left(b_{2}, \boldsymbol{b}\right)$, we define

$$
\begin{aligned}
& v_{21}=\int_{x=0}^{b_{2}-b_{1}} \int_{y=0}^{x} \tilde{r}_{2}^{\left(b_{2}\right)}(y) g_{21}(x-y) \mathrm{d} y\left(b_{2}-b_{1}-x\right) \mathrm{d} x, \\
& v_{20}=\int_{x=0}^{b_{2}-b_{1}} \tilde{r}_{2}^{\left(b_{2}\right)}(x)\left(b_{2}-b_{1}-x\right) \mathrm{d} x,
\end{aligned}
$$




$$
\begin{aligned}
B_{1, d, i} & =\int_{x=b_{2}-b_{1}}^{b_{2}} \int_{y=0}^{x} \tilde{r}_{2}^{\left(b_{2}\right)}(y) g_{21}(x-y) \mathrm{d} y v_{1, i}^{+}\left(b_{2}-x, b_{1}\right) \mathrm{d} x, \quad i=1,2, \\
B_{0, d, i}= & \int_{x=b_{2}-b_{1}}^{b_{2}} \tilde{r}_{2}^{\left(b_{2}\right)}(x) v_{1, i}^{+}\left(b_{2}-x, b_{1}\right) \mathrm{d} x, \quad i=1,2, \\
B_{1, u, 1}= & \int_{x=0}^{b_{2}-b_{1}} \int_{y=0}^{x} \tilde{r}_{2}^{\left(b_{2}\right)}(y) g_{21}(x-y) \mathrm{d} y \mathrm{~d} x, \\
B_{0, u, 1}= & \int_{x=0}^{b_{2}-b_{1}} \tilde{r}_{2}^{\left(b_{2}\right)}(x) \mathrm{d} x .
\end{aligned}
$$

Given that $R_{\boldsymbol{b}}(0)=b_{2}$ and $J(0)=2, v_{21}$ and $v_{20}$ are the expected discounted dividends paid when the first transition to state 1 occurs at time $\tilde{\mathcal{E}}_{2}$, with or without a claim arrival. The quantities $B_{1, d, i}$ and $B_{0, d, i}$ are the expected discounted times to reach level $b_{1}$, where, after the first transition to state 1 that occurs with or without a claim arrival at time $\tilde{\mathcal{E}}_{2}$, the surplus is below $b_{1}$. Similarly, $B_{1, u, i}$ and $B_{0, u, i}$ are the expected discounted times to reach $b_{1}$ when the first transition to state 1 with or without a claim arrival occurs when the surplus is above $b_{1}$ (and then drops to $b_{1}$ ).

Theorem 7.1. We have

$$
V_{1}\left(b_{1}, \boldsymbol{b}\right)=w_{1}^{\left(b_{1}\right)}+\sum_{j=1}^{2}\left(\sum_{k=1}^{2} A c_{1, k, j} D_{1}(1, k)+A_{1,2, j} D_{0}(1, k)\right) V_{j}\left(b_{1}, \boldsymbol{b}\right),
$$

where the coefficients are as defined by (4.5) with $b_{1}$ replacing $b$. For $b_{1} \leq u<b_{2}$,

$$
\begin{aligned}
V_{2}(u, \boldsymbol{b})= & D_{1}(2,1) v_{11}+D_{0}(2,1) v_{10}+\tilde{v}_{22}^{+}\left(u, b_{2}\right) V_{2}\left(b_{2}, \boldsymbol{b}\right) \\
& +\left(D_{1}(2,1)\left(\tilde{A}_{1, d, 1}(u)+\tilde{A}_{1, u, 1}(u)\right)\right. \\
& \left.+D_{0}(2,1)\left(\tilde{A}_{0, d, 1}(u)+\tilde{A}_{0, u, 1}(u)\right)\right) V_{1}\left(b_{1}, \boldsymbol{b}\right) \\
& +\left(D_{1}(2,1) \tilde{A}_{1, d, 2}(u)+D_{0}(2,1) \tilde{A}_{0, d, 2}(u)\right) V_{2}\left(b_{1}, \boldsymbol{b}\right), \\
V_{2}\left(b_{2}, \boldsymbol{b}\right)= & \tilde{w}_{2}+D_{1}(2,1) v_{21}+D_{0}(2,1) v_{20} \\
& +\left(D_{1}(2,1)\left(B_{1, d, 1}+B_{1, u, 1}\right)+D_{0}(2,1)\left(B_{0, d, 1}+B_{0, u, 1}\right)\right) V_{1}\left(b_{1}, \boldsymbol{b}\right) \\
& \left.+\left(D_{1}(2,1) B_{1, d, 2}+D_{0}(2,1) B_{0, d, 2}\right)\right) V_{2}\left(b_{1}, \boldsymbol{b}\right) .
\end{aligned}
$$

Substituting $u=b_{1}$ in (7.4) yields $V_{2}\left(b_{1}, \boldsymbol{b}\right)$.

Proof. Equation (7.3) for $V_{1}\left(b_{1}, \boldsymbol{b}\right)$ is the same as (4.6) for $i=1$, where $b$ is replaced by $b_{1}$. Equations (7.4) and (7.5) follow straightforwardly from the definitions of the coefficients defined in (7.1) and (7.2), respectively.

Remark 7.1. For $0<u \leq b_{1}$,

$$
V_{i}(u, \boldsymbol{b})=\sum_{j=1}^{2} v_{i, j}^{+}\left(u, b_{1}\right) V_{j}\left(b_{1}, \boldsymbol{b}\right), \quad i=1,2 .
$$

For $b_{1}<u$

$$
V_{1}(u, \boldsymbol{b})=u-b_{1}+V_{1}\left(b_{1}, \boldsymbol{b}\right) .
$$

For $b_{1} \leq u<b_{2}, V_{2}(u, \boldsymbol{b})$ is given by (7.4). For $u>b_{2}$,

$$
V_{2}(u, \boldsymbol{b})=u-b_{2}+V_{2}\left(b_{2}, \boldsymbol{b}\right) .
$$


7.1.2. The expected discounted penalty function. Let $\omega_{j}(u, \boldsymbol{b})$ be the expected discounted penalty function when $R_{b}(0)=u$ and $J(0)=j, j=1$, 2. For $0 \leq u \leq b_{1}$, let $v_{i j}^{-}\left(u, 0, b_{1}\right)$, $v_{i j}^{-}\left(u, z, b_{1}\right), \omega_{0, i}\left(u, b_{1}\right)$, and $\omega_{1,1}\left(b_{1}\right)$ be the same as $v_{i j}^{-}(u, 0), v_{i j}^{-}(u, z), \omega_{0, i}(u, b)$, and $\omega_{1,1}(b)$ in (4.8), (4.9), (4.11), and (4.12), where $b$ is replaced by $b_{1}$. Let

$$
\begin{gathered}
\tilde{v}_{22}^{\left(0, b_{2}\right)-}(u, 0)=\mathbb{E}_{2, u}\left[\mathrm{e}^{-\delta \tau_{0}^{-}} I\left(\tau_{0}^{-}<\tau_{b_{2}}^{+}, \tau_{0}^{-}<\tilde{\mathcal{E}}_{2}\right), \xi_{0}^{-}=0\right], \\
\tilde{v}_{22}^{\left(0, b_{2}\right)-}(u, z) \mathrm{d} z=\mathbb{E}_{2, u}\left[\mathrm{e}^{-\delta \tau_{0}^{-}} I\left(\tau_{0}^{-}<\tau_{b_{2}}^{+}, \tau_{0}^{-}<\tilde{\mathcal{E}}_{2}\right), \xi_{0}^{-} \in \mathrm{d} z\right] .
\end{gathered}
$$

We will obtain $\tilde{v}_{22}^{-}(u, 0)$ and $\tilde{v}_{22}^{-}(u, z)$ later. Similarly to $(4.11)$, define

$$
\begin{aligned}
\tilde{\omega}_{0,2}\left(u, b_{2}\right) & =\mathbb{E}_{2, u}\left[\mathrm{e}^{-\delta \tilde{\tau}_{0}^{-}} f\left(\xi_{0}^{-}\right) I\left(\tilde{\tau}_{0}^{-}<\tilde{\tau}_{b_{2}}^{+}, \tilde{\tau}_{0}^{-}<\tilde{\mathcal{E}}_{2}\right)\right] \\
& =\tilde{v}_{22}^{\left(0, b_{2}\right)-}(u, 0) f(0)+\int_{z=0}^{\infty} f(z) \tilde{v}_{22}^{\left(0, b_{2}\right)-}(u, z) \mathrm{d} z, \\
\tilde{\omega}_{1,2}\left(b_{2}\right) & =f(0) \int_{0}^{\infty} \mathrm{d} F_{T_{b_{2}}}(t) \exp \left(-\left(\eta_{2}+\delta\right) t\right)=f(0) \mathcal{L}_{T_{b_{2}}}\left(\eta_{2}+\delta\right),
\end{aligned}
$$

where $\tilde{\omega}_{0,2}\left(u, b_{2}\right)$ is the expected discounted penalty due to ruin occurrence before reaching $b_{2}$ and before state transition, given that $J(0)=2$, and $\tilde{\omega}_{1,2}\left(b_{2}\right)$ is the expected penalty function when ruin occurs due to diffusion before a state transition or claim arrival, and is defined similarly to (4.12) with $b_{2}$ replacing $b$. For $b_{1} \leq u<b_{2}$, let

$$
\begin{aligned}
P_{2,1, c, 0}(u) & =\int_{x=0}^{b_{2}} \tilde{u}_{21}^{\left(0, b_{2}\right)}(u, x) \int_{z=0}^{\infty} g_{21}(x+z) f(z) \mathrm{d} z, \\
P_{2,1, c}(u) & =\int_{x=0}^{b_{1}} \int_{y=x}^{b_{2}} \tilde{u}_{21}^{\left(0, b_{2}\right)}(u, y) g_{21}(y-x) \mathrm{d} y \omega_{0,1}\left(x, b_{1}\right) \mathrm{d} x, \\
P_{2,1,0}(u) & =\int_{0}^{b_{1}} \tilde{u}_{21}^{\left(0, b_{2}\right)}(u, x) \omega_{0,1}\left(x, b_{1}\right),
\end{aligned}
$$

where

- $P_{2,1, c, 0}(u)$ is the expected discounted penalty when ruin occurs due to a claim arrival with a transition from state 2 to 1 ,

- $P_{2,1, c}(u)$ and $P_{2,1,0}(u)$ are the expected discounted penalties when a transition to state 1 , with or, respectively, without a claim arrival, occurs before ruin, and the surplus after that transition is less than $b_{1}$, with ruin then occurring before reaching $b_{1}$.

For $R_{b}(0)=b_{2}$ and $J(0)=2$, we will apply the following expressions for the expected discounted penalty when ruin occurs before paying dividends:

$$
\begin{aligned}
P_{1, k} & =\int_{0}^{b_{2}} r_{2}^{\left(b_{2}\right)}(x) g_{2 k}\left(b_{2}-x+z\right) f(z) \mathrm{d} z, \quad k=1,2, \\
P_{2, k} & =\int_{x=b_{2}-b_{1}}^{b_{2}} \int_{y=0}^{x} r_{2}^{\left(b_{2}\right)}(y) g_{2, k}(x-y) \mathrm{d} y \omega_{0, k}\left(b_{2}-x, b_{1}\right) \mathrm{d} x, \quad k=1,2, \\
P_{3} & =\int_{x=b_{2}-b_{1}}^{b_{2}} r_{2}^{\left(b_{2}\right)}(x) \omega_{0,1}\left(b_{2}-x, b_{1}\right) \mathrm{d} x,
\end{aligned}
$$




$$
\begin{aligned}
P_{4}= & \int_{x=0}^{b_{2}-b_{1}} \int_{y=0}^{x} r_{2}^{\left(b_{2}\right)}(y) g_{22}(x-y) \mathrm{d} y \tilde{\omega}_{0,2}\left(b_{2}-x, b_{2}\right) \mathrm{d} x \\
P_{5}= & \int_{x=0}^{b_{2}-b_{1}} \int_{y=0}^{x} r_{2}^{\left(b_{2}\right)}(y) g_{22}(x-y) \mathrm{d} y \\
& \times \int_{v=0}^{b_{1}} D_{0}(2,1) \tilde{u}_{21}^{\left(0, b_{2}\right)}\left(b_{2}-x, v\right) \omega_{0,1}\left(v, b_{1}\right) \mathrm{d} v \mathrm{~d} x \\
P_{6}= & \int_{x=0}^{b_{2}-b_{1}} \int_{y=0}^{x} r_{2}^{\left(b_{2}\right)}(y) g_{22}(x-y) \mathrm{d} y \\
& \times \int_{v=0}^{b_{1}} D_{1}(2,1) \tilde{u}_{21}^{\left(0, b_{2}\right)}\left(b_{2}-x, v\right) \int_{z=0}^{\infty} g_{21}(v+z) f(z) \mathrm{d} z \mathrm{~d} v \mathrm{~d} x \\
P_{7}= & \int_{x=0}^{b_{2}-b_{1}} \quad \int_{y=0}^{x} r_{2}^{\left(b_{2}\right)}(y) g_{22}(x-y) \mathrm{d} y \\
& \times \int_{v=0}^{b_{1}} D_{1}(2,1) \tilde{u}_{21}^{\left(0, b_{2}\right)}\left(b_{2}-x, v\right) \int_{z=0}^{v} g_{21}(z) \omega_{0,1}\left(v-z, b_{1}\right) \mathrm{d} z \mathrm{~d} v \mathrm{~d} x .
\end{aligned}
$$

Here

- $P_{1, k}, k=1,2$, are the discounted penalties when ruin occurs due to a claim arrival with a state transition,

- $P_{2, k}, k=1,2$, and $P_{3}$ are the expected discounted penalties when a state transition, with or, respectively, without a claim arrival, occurs at time $\varepsilon_{2}$ before ruin, and the surplus process after the state transition is between 0 and $b_{1}$, with ruin then occurring before reaching the level $b_{1}$,

- $P_{4}$ is the expected discounted penalty when an arrival at state 2 occurs before ruin at time $\varepsilon_{2}$, and the surplus after the arrival is between $b_{1}$ and $b_{2}$, with ruin then occurring before reaching the level $b_{2}$ and before a transition to state 1 ,

- $P_{i}, i=5,6,7$, are the expected discounted penalties when an arrival at state 2 occurs before ruin at time $\varepsilon_{2}$, and the surplus after the arrival is between $b_{1}$ and $b_{2}$, then a transition to state 1 without $\left(P_{5}\right)$ or with $\left(P_{6}, P_{7}\right)$ an arrival occurs before reaching $b_{2}$ and before ruin, when the process level is less than $b_{1}$. In (7.6) the arrival causes an immediate ruin. In (7.7) ruin occurs after that arrival and before the process reaches $b_{1}$.

Finally, we consider the following discounted exit times $\left(R_{\boldsymbol{b}}(0)=b_{2}\right.$ and $\left.J(0)=2\right)$ :

$$
\begin{aligned}
d_{1, k, j} & =\int_{x=b_{2}-b_{1}}^{b_{2}} \int_{y=0}^{x} r_{2}^{\left(b_{2}\right)}(y) g_{2, k}(x-y) \mathrm{d} y v_{k j}^{+}\left(b_{2}-x, b_{1}\right) \mathrm{d} x, \quad k, j=1,2, \\
d_{2, j} & =\int_{x=b_{2}-b_{1}}^{b_{2}} r_{2}^{\left(b_{2}\right)}(x) v_{1 j}^{+}\left(b_{2}-x, b_{1}\right) \mathrm{d} x, \quad j=1,2, \\
d_{3} & =\int_{x=0}^{b_{2}-b_{1}} \int_{y=0}^{x} r_{2}^{\left(b_{2}\right)}(y) g_{22}(x-y) \tilde{v}_{22}^{\left(0, b_{2}\right)+}\left(b_{2}-x, b_{2}\right) \mathrm{d} y \mathrm{~d} x, \\
d_{4} & =\int_{x=0}^{b_{2}-b_{1}} \int_{y=0}^{x} r_{2}^{\left(b_{2}\right)}(y) g_{21}(x-y) \mathrm{d} y \mathrm{~d} x,
\end{aligned}
$$




$$
\begin{aligned}
d_{5}= & \int_{x=0}^{b_{2}-b_{1}} r_{2}^{\left(b_{2}\right)}(x) \mathrm{d} x \\
d_{6, j}= & D_{0}(2,1) \int_{x=0}^{b_{2}-b_{1}} \int_{y=0}^{x} r_{2}^{\left(b_{2}\right)}(y) g_{22}(x-y) \mathrm{d} y \\
& \times \int_{v=0}^{b_{1}} \tilde{u}_{21}^{\left(0, b_{2}\right)}\left(b_{2}-x, v\right) \mathrm{d} x v_{1 j}^{+}\left(v, b_{1}\right) \mathrm{d} v, \quad j=1,2, \\
d_{7, j}= & D_{1}(2,1) \int_{x=0}^{b_{2}-b_{1}} \int_{y=0}^{x} r_{2}^{\left(b_{2}\right)}(y) g_{22}(x-y) \mathrm{d} y \\
& \times \int_{v=0}^{b_{1}} \int_{z=v}^{b_{2}} \tilde{u}_{21}^{\left(0, b_{2}\right)}\left(b_{2}-x, z\right) \mathrm{d} x g_{21}(z-v) \mathrm{d} z v_{1 j}^{+}\left(v, b_{1}\right) \mathrm{d} v, \quad j=1,2, \\
d_{8}= & D_{0}(2,1) \int_{x=0}^{b_{2}-b_{1}} \int_{y=0}^{x} r_{2}(y) g_{22}(x-y) \mathrm{d} y \int_{v=b_{1}}^{b_{2}} \tilde{u}_{21}^{\left(0, b_{2}\right)}\left(b_{2}-x, v\right) \mathrm{d} v \mathrm{~d} x, \\
d_{9}= & D_{1}(2,1) \int_{x=0}^{b_{2}-b_{1}} \int_{y=0}^{x} r_{2}^{\left(b_{2}\right)}(y) g_{22}(x-y) \mathrm{d} y \\
& \times \int_{v=b_{1}}^{b_{2}} \int_{z=v}^{b_{2}} \tilde{u}_{21}^{\left(0, b_{2}\right)}\left(b_{2}-x, z\right) g_{21}(z-v) \mathrm{d} z \mathrm{~d} v \mathrm{~d} x .
\end{aligned}
$$

Here

- $d_{1, k, j}$ and $d_{2, j}$ are the discounted times to reach level $b_{1}$ when the modulated state is $j$, when a transition to state $k$ occurs at time $\varepsilon_{2}$ before ruin, with or, respectively, without a claim arrival, and the surplus after the transition is less than $b_{1}$,

- when the first transition at time $\varepsilon_{2}$ is a claim arrival in state 2 , and the surplus after the arrival is between $b_{1}$ and $b_{2}, d_{3}$ is the discounted time to reach $b_{2}$ before ruin and before transition to state 1 ,

- $d_{4}$ and $d_{5}$ are the discounted times of the first transition to state 1 at time $\mathcal{E}_{2}$, with or, respectively, without a claim arrival, and the surplus after the transition is between $b_{1}$ and $b_{2}$ and drops to $b_{1}$,

- $d_{6, j}, d_{7, j}, j=1,2, d_{8}$, and $d_{9}$ are the discounted times to reach $b_{1}$ before ruin, where the first transition at time $\varepsilon_{2}$ is a claim at state 2 and the surplus after that transition is between $b_{1}$ and $b_{2}$.

In $d_{6, j}$ and $d_{7, j}, j=1,2$, the process level after the transition is below $b_{1}$, and so we consider the discounted time to reach $b_{1}$, while in $d_{8}$ and $d_{9}$ the process reaches state 1 when it is above $b_{1}$, and then drops to $b_{1}$.

We present now the linear equations for $\omega_{i}\left(b_{j}, \boldsymbol{b}\right)$.

Proposition 7.1. Similarly to (4.13)-(4.17) we have

$$
\begin{aligned}
\omega_{1}\left(b_{1}, \boldsymbol{b}\right)= & \omega_{1,1}\left(b_{1}\right)+\sum_{k=1}^{2} D_{1}(1, k) \int_{x=0}^{b_{1}} \int_{z=0}^{\infty} r_{1}^{\left(b_{1}\right)}(x) g_{1 k}\left(b_{1}-x+z\right) f(z) \mathrm{d} z \mathrm{~d} x \\
& +\sum_{k=1}^{2} D_{1}(1, k) \int_{x=0}^{b_{1}} \int_{y=0}^{x} r_{1}^{\left(b_{1}\right)}(y) g_{1 k}(x-y) \mathrm{d} y \omega_{0, k}\left(b_{1}-x, b_{1}\right) \mathrm{d} x
\end{aligned}
$$




$$
\begin{aligned}
& +D_{0}(1,2) \int_{x=0}^{b_{1}} r_{1}^{\left(b_{1}\right)}(x) \omega_{0,2}\left(b_{1}-x, b_{1}\right) \mathrm{d} x \\
& +\sum_{j=1}^{2} \sum_{k=1}^{2} D_{1}(1, k) A c_{1, k, j} \omega_{j}\left(b_{1}, \boldsymbol{b}\right)+\sum_{j=1}^{2} \sum_{k=1}^{2} D_{0}(i, k) A_{1, k, j} \omega_{j}\left(b_{1}, \boldsymbol{b}\right),
\end{aligned}
$$

where the coefficients are as defined in (4.5) with $b_{1}$ replacing $b$.

For $b_{1} \leq u<b_{2}$,

$$
\begin{aligned}
\omega_{2}(u, \boldsymbol{b})= & D_{1}(2,1)\left(P_{2,1, c, 0}(u)+P_{2,1, c}(u)\right)+D_{0}(2,1) P_{2,1,0}(u) \\
& +\tilde{v}_{22}^{\left(0, b_{2}\right)+}\left(u, b_{2}\right) \omega_{2}\left(b_{2}, \boldsymbol{b}\right)+\left(D_{1}(2,1)\left(\tilde{A}_{1, d, 1}(u)+\tilde{A}_{1, u, 1}(u)\right)\right. \\
& \left.+D_{0}(2,1)\left(\tilde{A}_{0, d, 1}(u)+\tilde{A}_{0, u, 1}(u)\right)\right) \omega_{1}\left(b_{1}, \boldsymbol{b}\right) \\
& +\left(D_{1}(2,1) \tilde{A}_{1, d, 2}(u)+D_{0}(2,1) \tilde{A}_{0, d, 2}(u)\right) \omega_{2}\left(b_{1}, \boldsymbol{b}\right) \\
\omega_{2}\left(b_{2}, \boldsymbol{b}\right)= & \tilde{\omega}_{1,2}\left(b_{2}\right)+\sum_{k=1}^{2} D_{1}(2, k)\left(P_{1, k}+P_{2, k}\right)+D_{0}(2,1) P_{3} \\
+ & D_{1}(2,2)\left(P_{4}+P_{5}+P_{6}+P_{7}\right) \\
& +\left[\sum_{k=1}^{2} D_{1}(2, k) d_{1, k, 1}+D_{0}(2,1) d_{2,1}+D_{1}(2,1) d_{4}+D_{0}(2,1) d_{5}\right. \\
& \left.+D_{1}(2,2)\left(d_{6,1}+d_{7,1}+d_{8}+d_{9}\right)\right] \omega_{1}\left(b_{1}, \boldsymbol{b}\right) \\
& +\left[\sum_{k=1}^{2} D_{1}(2, k) d_{1, k, 2}+D_{0}(2,1) d_{2,2}+D_{1}(2,2)\left(d_{6,2}+d_{7}\right)\right] \omega_{2}\left(b_{1}, \boldsymbol{b}\right) \\
& +D_{1}(2,2) d_{3} \omega_{2}\left(b_{2}, \boldsymbol{b}\right) .
\end{aligned}
$$

By substituting $u=b_{1}$ in (7.8) we obtain the equations for $\omega_{2}\left(b_{1}, \boldsymbol{b}\right)$.

Proof. The proof straightforwardly follows from the definitions of the coefficients.

It remains to derive $\tilde{v}_{22}^{-}(u, 0)$ and $\tilde{v}_{22}^{-}(u, z)$ for $0<u<b_{2}$. Let $\Phi_{2}\left(\delta+\lambda_{21}\right)$ be the largest solution of $\varphi_{2}(\alpha)=\delta+\lambda_{21}$. Applying the change-of-measure formula for the process $\tilde{X}_{2}$, yields

$$
\begin{gathered}
\tilde{v}_{22}^{\left(0, b_{2}\right)-}(u, z) \mathrm{d} z=\mathrm{e}^{\Phi_{2}\left(\delta+\lambda_{21}\right)(u+z)} \mathbb{P}^{\Phi\left(\delta+\lambda_{21}\right)}\left(\tilde{\tau}_{0}^{-}<\tilde{\tau}_{b_{2}}^{+},-\tilde{X}_{2}\left(\tilde{\tau}_{0}^{-}\right) \in \mathrm{d} z \mid \tilde{X}_{2}=u\right), \\
\tilde{v}_{22}^{\left(0, b_{2}\right)-}(u, 0)=\mathrm{e}^{\Phi_{2}\left(\delta+\lambda_{21}\right) u} \mathbb{P}^{\Phi\left(\delta+\lambda_{21}\right)}\left(\tilde{\tau}_{0}^{-}<\tilde{\tau}_{b_{2}}^{+}, \tilde{X}_{2}\left(\tilde{\tau}_{0}^{-}\right)=0 \mid \tilde{X}_{2}=u\right) .
\end{gathered}
$$

Under $\mathbb{P}^{\Phi\left(\delta+\lambda_{21}\right)}, \tilde{X}_{2}$ is a Lévy process with negative phase-type jumps with representation $\left(\tilde{\boldsymbol{\pi}}_{22}, \tilde{\boldsymbol{T}}_{22}\right)$, and Lévy exponent

$$
\tilde{\varphi}_{2}(\alpha)=\tilde{c}_{2} \alpha+\frac{\sigma^{2} \alpha^{2}}{2}+\tilde{\beta}_{2}\left(\left(\alpha \boldsymbol{I}+\tilde{T}_{22}\right)^{-1} \tilde{\boldsymbol{t}}_{22}-1\right),
$$

where $\tilde{\boldsymbol{\pi}}_{22}, \tilde{\boldsymbol{T}}_{22}$, and $\tilde{\boldsymbol{t}}_{22}$ are as given in Lemma 4.1, and $\tilde{c}_{2}$ is as given in (P1). Let $\tilde{X}_{2}^{f}$ be the fluid version of $\tilde{X}_{2}$ under $\mathbb{P}^{\Phi\left(\delta+\lambda_{21}\right)}$. The process $\tilde{X}_{2}^{f}$ is a MAP with $m_{22}+1$ states and modulating process $\tilde{J}_{2}^{f}$. When in state $0, \tilde{X}_{2}^{f}$ evolves as a Brownian motion with drift $\tilde{c}_{2}$, and, 
when in state $k, k=1, \ldots, m_{22}+1$, it is a linear line with slope -1 . State $k$ corresponds to phase $k$ of the claim. Let $\tilde{\boldsymbol{\pi}}_{22}, \tilde{\boldsymbol{T}}_{22}$, and $\tilde{\boldsymbol{t}}_{22}$ be as defined in (4.3). Similarly to the definition of $\boldsymbol{F}$ in Section 5, the cumulant matrix for $\tilde{X}_{2}^{f}$ under $\mathbb{P}^{\Phi\left(\delta+\lambda_{21}\right)}$ is $\boldsymbol{F}_{2}(\alpha)$, where

$$
\boldsymbol{F}_{2}(\alpha)=\left(\begin{array}{cc}
-\tilde{\beta}_{2} & \tilde{\boldsymbol{\pi}}_{22} \\
\tilde{\boldsymbol{t}}_{22} & \tilde{\boldsymbol{T}}_{22}
\end{array}\right)+\operatorname{diag}\left(\tilde{\varphi}_{2}(\alpha),-\alpha, \ldots,-\alpha\right) .
$$

Define

$$
\begin{aligned}
& \tilde{\gamma}^{+}(u)=\mathbb{P}^{\Phi\left(\delta+\lambda_{21}\right)}\left(\tilde{\tau}_{b_{2}}^{f+}<\tilde{\tau}_{0}^{f-} \mid \tilde{X}_{2}^{f}(0)=u\right), \\
& \tilde{\gamma}_{k}^{-}(u)=\mathbb{P}^{\Phi\left(\delta+\lambda_{21}\right.}\left(\tilde{\tau}_{0}^{f-}<\tilde{\tau}_{b_{2}}^{f+}, J^{f}\left(\tilde{\tau}_{0}^{f-}\right)=k \mid \tilde{X}_{2}^{f}(0)=u\right),
\end{aligned}
$$

where $\tilde{\tau}_{b_{2}}^{f+}$ and $\tilde{\tau}_{0}^{f-}$ are first times that $\tilde{X}_{2}^{f}$ hits $b_{2}$ and 0 , respectively. To find the probabilities $\tilde{\gamma}_{k}^{-}(u)$, we apply the same technique as described in (4.4). Assume that $\left|\boldsymbol{F}_{2}(\alpha)\right|=0$ has $m_{22}+2$ different roots $\alpha_{r}, r=1, \ldots, m_{22}+2$, and let $\boldsymbol{h}^{2 f}\left(\alpha_{r}\right)$ be the corresponding right eigenvectors. Then the following equations hold for $\tilde{\gamma}_{k}^{-}(u), k=0, \ldots, m_{22}, \tilde{\gamma}^{+}(u)$ :

$$
\mathrm{e}^{\alpha_{r} u} h_{0}^{2 f}\left(\alpha_{r}\right)=\mathrm{e}^{\alpha_{r} b_{2}} \tilde{\gamma}^{+}(u)+\sum_{j=0}^{m_{22}} \tilde{\gamma}_{k}^{-}(u) h_{j}^{2 f}\left(\alpha_{r}\right), \quad r=1, \ldots, m_{22}+2 .
$$

Let $\tilde{\boldsymbol{\gamma}}^{-}(u)=\left(\gamma_{1}^{-}(u), \ldots, \tilde{\gamma}_{m_{22}}^{-}(u)\right)$. Then

$$
\begin{gathered}
\mathbb{P}^{\Phi\left(\delta+\lambda_{21}\right)}\left(\tilde{\tau}_{0}^{-}<\tilde{\tau}_{b_{2}}^{+},-\tilde{X}_{2}\left(\tilde{\tau}_{0}^{-}\right) \in \mathrm{d} z \mid \tilde{X}_{2}(0)=u\right)=\tilde{\boldsymbol{\gamma}}^{-}(u) \mathrm{e}^{\tilde{T}_{22} z} \tilde{\boldsymbol{t}}_{22} \mathrm{~d} z \\
\mathbb{P}^{\Phi\left(\delta+\lambda_{21}\right)}\left(\tilde{\tau}_{0}^{-}<\tilde{\tau}_{b_{2}}^{+},-\tilde{X}_{2}\left(\tilde{\tau}_{0}^{-}\right)=0 \mid \tilde{X}_{2}(0)=u\right)=\tilde{\gamma}_{0}^{-}(u) .
\end{gathered}
$$

Applying the change of measure we obtain

$$
\tilde{v}_{22}^{\left(0, b_{2}\right)-}(u, z)=\mathrm{e}^{\Phi\left(\delta+\lambda_{21}\right)(u+z)} \tilde{\boldsymbol{\gamma}}^{-}(u) \mathrm{e}^{\tilde{\boldsymbol{T}}_{22} z} \tilde{\boldsymbol{t}}_{22}, \quad \tilde{\boldsymbol{v}}_{22}^{\left(0, b_{2}\right)-}(u, 0)=\mathrm{e}^{\Phi\left(\delta+\lambda_{21}\right) u} \tilde{\gamma}_{0}^{-}(u) .
$$

\subsection{A barrier strategy dependent on the environmental state of the dual model}

In the dual model there are only negative claims, i.e. positive jumps. Given that $J(0)=2$ and $R_{\boldsymbol{b}}(0)=u, b_{1}<u<b_{2}$, then until time $\tilde{\mathcal{E}}_{2}$ the process behaves as a Lévy process $X_{2}^{+}(t)$ with positive jumps, i.e.

$$
X_{2}^{+}(t)=u+\sigma_{2} B(t)+S_{2}(t)
$$

where $S_{2}$ is a compound Poisson process with arrival rate $\beta_{2}$, i.i.d. phase-type jumps with representation $\left(\boldsymbol{\pi}_{22}^{+}, \boldsymbol{T}_{22}^{+}\right)$, and Lévy exponent

$$
\varphi_{2}(\alpha)=c_{2} \alpha+\frac{\sigma_{2}^{2} \alpha^{2}}{2}+\beta_{2}\left(\left(-\alpha \boldsymbol{I}-\boldsymbol{T}_{22}^{+}\right)^{-1}-1\right) .
$$

Let $\Phi_{2}\left(\alpha+\lambda_{21}\right)$ be the largest solution of $\varphi_{2}(\alpha)=\lambda_{21}+\delta$. Let

$$
\tilde{\tau}_{a}^{+}=\inf \left\{t: X_{2}^{+}(t) \geq a\right\}, \quad \tilde{\tau}_{a}^{-}=\inf \left\{t: X_{2}^{+}(t) \leq a\right\} .
$$

For $x<u<y$, define

$$
\begin{gathered}
\tilde{v}_{22}^{(x, y)+}(u, z) \mathrm{d} z=\mathbb{E}\left(\mathrm{e}^{-\delta \tilde{x}_{y}^{+}} I\left(\tilde{\tau}_{y}^{+}<\tilde{\tau}_{x}^{-}, \tilde{\tau}_{y}^{+}<\tilde{\mathcal{E}}_{2}, X_{2}^{+}\left(\tilde{\tau}_{y}^{+}\right) \in y+\mathrm{d} z\right) \mid X_{2}^{+}(0)=u\right), \\
\tilde{v}_{22}^{(x, y)+}(u, 0)=\mathbb{E}\left(\mathrm{e}^{-\delta \tilde{\tau}_{y}^{+}} I\left(\tilde{\tau}_{y}^{+}<\tilde{\tau}_{x}^{-}, \tilde{\tau}_{y}^{+}<\tilde{\mathcal{E}}_{2}, X_{2}^{+}\left(\tilde{\tau}_{y}^{+}\right)=y\right) \mid X_{2}^{+}(0)=u\right), \\
\tilde{v}_{22}^{(x, y)-}(u, x)=\mathbb{E}\left(\mathrm{e}^{-\delta \tilde{\tau}_{x}^{-}} I\left(\tilde{\tau}_{x}^{-}<\tilde{\tau}_{y}^{+}, \tilde{\tau}_{x}^{-}<\tilde{\mathcal{E}}_{2}, X_{2}^{+}\left(\tilde{\tau}_{x}^{-}\right)=x\right) \mid X_{2}^{+}(0)=u\right) .
\end{gathered}
$$


Applying the change-of-measure formula we obtain

$$
\begin{aligned}
\tilde{v}_{22}^{(x, y)+}(u, z) \mathrm{d} z & =\mathrm{e}^{\Phi\left(\delta+\lambda_{21}\right)(u-y-z)} \tilde{\gamma}_{22}^{(x, y)+}(u, z) \mathrm{d} z, \\
\tilde{v}_{22}^{(x, y)+}(u, 0) & =\mathrm{e}^{\Phi\left(\delta+\lambda_{21}\right)(u-y)} \tilde{\gamma}_{22}^{(x, y)+}(u, 0), \\
\tilde{v}_{22}^{(x, y)-}(u) & =\mathrm{e}^{\Phi\left(\delta+\lambda_{21}\right)(u-x)} \tilde{\gamma}_{22}^{(x, y)-}(u, x),
\end{aligned}
$$

where

$$
\begin{aligned}
\tilde{\gamma}_{22}^{(x, y)+}(u, z) \mathrm{d} z & =\mathbb{P}^{\Phi\left(\delta+\lambda_{21}\right)}\left(\tilde{\tau}_{y}^{+}<\tilde{\tau}_{x}^{-}, X_{2}^{+}\left(\tilde{\tau}_{y}^{+}\right) \in y+\mathrm{d} z \mid X_{2}^{+}(0)=u\right), \\
\tilde{\gamma}_{0}^{(x, y)+}(u, y) & =\mathbb{P}^{\Phi\left(\delta+\lambda_{21}\right)}\left(\tilde{\tau}_{y}^{+}<\tilde{\tau}_{x}^{-}, X_{2}^{+}\left(\tilde{\tau}_{y}^{+}\right)=y \mid X_{2}^{+}(0)=u\right), \\
\tilde{\gamma}_{22}^{(x, y)-}(u, x) & =\mathbb{P}^{\Phi\left(\delta+\lambda_{21}\right)}\left(\tilde{\tau}_{x}^{-}<\tilde{\tau}_{y}^{+}, X_{2}^{+}\left(\tilde{\tau}_{x}^{-}\right)=x \mid X_{2}^{+}(0)=u\right) .
\end{aligned}
$$

Let

$$
\tilde{v}_{22}^{(x, y)+}(u, \cdot)=\tilde{v}_{22}^{(x, y)+}(u, 0)+\int_{0}^{\infty} \tilde{v}_{22}^{(x, y)+}(u, z) \mathrm{d} z .
$$

Let $\tilde{\boldsymbol{\pi}}_{22}^{+}, \tilde{\boldsymbol{T}}_{22}^{+}$, and $\tilde{\boldsymbol{t}}_{22}^{+}$be the parameters of the phase-type distribution for the claim amount under $\mathbb{P}^{\Phi\left(\delta+\lambda_{21}\right)}$, as defined in (4.3), and let $\tilde{\varphi}_{2}(\alpha)$ be the Lévy exponent of $X_{2}^{+}$under $\mathbb{P}^{\Phi\left(\delta+\lambda_{21}\right)}$. To obtain (7.10)-(7.12), we resort to the fluid model of $X_{2}^{+}$, where upward jumps are replaced by lines with slope 1 . The generator for the fluid MAP (under $\left.\mathbb{P}^{\Phi\left(\delta+\lambda_{21}\right)}\right)$ is

$$
\boldsymbol{Q}_{22}^{+}=\left(\begin{array}{cc}
-\tilde{\beta}_{2} & \tilde{\beta}_{2} \tilde{\boldsymbol{\pi}}_{22}^{+} \\
\tilde{\boldsymbol{t}}_{22}^{+} & \tilde{\boldsymbol{T}}_{22}^{+}
\end{array}\right)
$$

Let $\boldsymbol{F}_{22}^{+}(\alpha)=\boldsymbol{Q}_{22}^{+}+\operatorname{diag}\left(\tilde{\varphi}_{2}(\alpha), \alpha, \ldots, \alpha\right)$. Assume that the equation $\left.\mid \boldsymbol{F}_{22}^{+}(\alpha)\right) \mid=0$ has $m_{22}+2$ different roots $\alpha_{i}, i=1, \ldots, m_{22}+2$. Let $\boldsymbol{h}^{f}\left(\alpha_{r}\right)$ be the eigenvector of $\boldsymbol{F}_{22}^{+}\left(\alpha_{r}\right)$. Consider the fluid model of the process under $\mathbb{P}^{\Phi\left(\delta+\lambda_{21}\right)}$. Let $\tilde{\gamma}_{0}^{(x, y)-}(u)$ be the probability that the process hits $x$ before $y$ (by diffusion), let $\tilde{\gamma}_{0}^{(x, y)+}(u)$ be the probability that it hits $y$ before $x$ by diffusion, and let $\tilde{\gamma}_{i}^{(x, y)+}(u), i=1, \ldots, m_{22}$, be the probabilities that the process hits $y$ by a claim at phase $i$ (before $x$ ). These probabilities are the solutions to the equations

$$
\mathrm{e}^{\alpha_{r} u} h_{0}^{f}\left(\alpha_{r}\right)=\mathrm{e}^{\alpha_{r} y} \sum_{i=0}^{m_{22}} h_{i}^{f}\left(\alpha_{r}\right) \tilde{\gamma}_{i}^{(x, y)+}(u)+\mathrm{e}^{\alpha_{r} x} \tilde{\gamma}_{0}^{(x, y)-}(u) .
$$

Let $\tilde{\boldsymbol{\gamma}}^{(x, y)+}(u)=\left(\tilde{\gamma}_{1}^{(x, y)+}(u), \ldots, \tilde{\gamma}_{m_{22}}^{(x, y)+}(u)\right)$. Then

$$
\tilde{\gamma}_{22}^{(x, y)+}(u, z)=\tilde{\boldsymbol{\gamma}}^{(x, y)+}(u) \mathrm{e}^{\tilde{\boldsymbol{T}}_{22}^{+} z} \tilde{\boldsymbol{t}}_{22}^{+} .
$$

Let

$$
\lambda_{21} \tilde{u}_{21}^{(a, b)+}(x, y) \mathrm{d} y=\mathbb{E}\left[\mathrm{e}^{-\delta \tilde{\mathcal{E}}_{2}} I\left(\tilde{\mathcal{E}}_{2}<\tilde{\tau}_{a}^{-} \wedge \tilde{\tau}_{b}^{+}, X_{2}^{+}\left(\tilde{\mathcal{E}}_{2}\right) \in \mathrm{d} y\right) \mid X_{2}^{+}(0)=x\right] .
$$

Let $\tilde{W}$ be the scale function of $-X_{2}^{+}$. By Theorem 8.7 of Kyprianou (2006) (adapted to processes with positive jumps),

$$
\tilde{u}_{21}^{(a, b)+}(x, y)=\frac{\tilde{W}_{2}^{\left(\delta+\lambda_{21}\right)}(y-a) \tilde{W}_{2}^{\left(\delta+\lambda_{21}\right)}(b-x)}{\tilde{W}_{2}^{\left(\delta+\lambda_{21}\right)}(b-a)}-\tilde{W}_{2}^{\left(\delta+\lambda_{21}\right)}(y-x) .
$$


For $b_{1}<u<b_{2}$ and $J(0)=2$, let

$$
\begin{aligned}
& \ell_{1}(u)=\int_{x=b_{1}}^{\infty} \int_{y=b_{1}}^{x \wedge b_{2}} D_{1}(2,1) \tilde{u}_{21}^{\left(b_{1}, b_{2}\right)+}(u, y) g_{21}^{+}(x-y) \mathrm{d} y\left(x-b_{1}\right) \mathrm{d} x, \\
& \ell_{2}(u)=D_{0}(2,1) \int_{x=b_{1}}^{b_{2}} \tilde{u}_{21}^{\left(b_{1}, b_{2}\right)+}(u, x)\left(x-b_{1}\right) \mathrm{d} x \\
& \ell_{3}(u)=\int_{z=0}^{\infty} \tilde{v}_{22}^{\left(b_{1}, b_{2}\right)+}(u, z) z \mathrm{~d} z .
\end{aligned}
$$

Let

$$
\ell(u)=\ell_{1}(u)+\ell_{2}(u)+\ell_{3}(u),
$$

where $\ell_{1}$ and $\ell_{2}$ describe the expected discounted dividends before the process drops to $b_{1}$ due to a transition to state 1 that occurs when the surplus is above $b_{1}$, and $\ell_{3}$ is the expected discounted dividends when the process reaches $b_{2}$ before $b_{1}$ and before a transition to state 1 . Let

$$
L(u)=\lambda_{21} \int_{y=b_{1}}^{b_{2}} \tilde{u}_{21}^{\left(b_{1}, b_{2}\right)+}(u, y) \mathrm{d} y
$$

be the expected discounted time to transition to state 1 before reaching $b_{1}$ or $b_{2}$. Define $v_{i j}^{+}\left(u, z, b_{1}\right)$ similarly to $v_{i, j}^{+}(u, z)$ in (5.1a). For $0<u<b_{1}$, we define the following possible dividend payments and exit times:

$$
\begin{aligned}
& d_{1}^{+}(u)=\int_{z=0}^{\infty} g_{11}^{+}\left(b_{1}-u+z\right) z \mathrm{~d} z, \\
& d_{2}^{+}(u)=\int_{z=b_{2}-b_{1}}^{\infty} g_{12}^{+}\left(b_{1}-u+z\right)\left(z-b_{2}+b_{1}\right) \mathrm{d} z, \\
& d_{3}^{+}(u)=\int_{z=0}^{b_{2}-b_{1}} g_{12}^{+}\left(b_{1}-u+z\right) \ell\left(b_{1}+z\right) \mathrm{d} z, \quad i=1,2, \\
& d_{4, i}^{+}(u)=\int_{z=0}^{\infty} v_{i 1}^{+}\left(u, z, b_{1}\right) z \mathrm{~d} z, \quad i=1,2, \\
& d_{5, i}^{+}(u)=\int_{z=b_{2}-b_{1}}^{\infty} v_{i 2}^{+}\left(u, z, b_{1}\right)\left(z-b_{2}+b_{1}\right) \mathrm{d} z, \\
& d_{6, i}^{+}(u)=\int_{z=0}^{b_{2}-b_{1}} v_{i 2}^{+}\left(u, z, b_{1}\right) \ell\left(b_{1}+z\right) \mathrm{d} z .
\end{aligned}
$$

For $0<u<b_{1}$, we now define the following discounted exit times:

$$
\begin{aligned}
& \chi_{2, i}(u)=\int_{z=b_{2}-b_{1}}^{\infty} v_{i, 2}^{+}\left(u, z, b_{1}\right) \mathrm{d} z, \quad i=1,2, \\
& \chi_{3, i}(u)=\int_{z=0}^{b_{2}-b_{1}} v_{i, 2}^{+}\left(u, z, b_{1}\right) \tilde{v}_{22}^{\left(b_{1}, b_{2}\right)+}\left(b_{1}+z, \cdot\right) \mathrm{d} z, \\
& \chi_{4, i}(u)=\int_{z=0}^{b_{2}-b_{1}} v_{i, 2}^{+}\left(u, z, b_{1}\right) L\left(b_{1}+z\right) \mathrm{d} z, \quad i=1,2, \\
& \chi_{5, i}(u)=\int_{z=0}^{b_{2}-b_{1}} v_{i, 2}^{+}\left(u, z, b_{1}\right) \tilde{v}_{22}^{\left(b_{1}, b_{2}\right)-}\left(b_{1}+z\right) \mathrm{d} z+v_{i, 2}^{+}\left(u, 0, b_{1}\right), \quad i=1,2 .
\end{aligned}
$$


For $R_{\boldsymbol{b}}(0)=b_{1}$ and $J(0)=1$, we define the following dividends payed due to negative claims (positive jumps), before hitting one of the barriers:

$$
\begin{aligned}
\vartheta_{1}^{+} & =\int_{x=0}^{b_{1}} r_{1}^{\left(b_{1}\right)}(x) d_{1}^{+}\left(b_{1}-x\right) \mathrm{d} x, \\
\vartheta_{2}^{+} & =\int_{x=0}^{b_{1}} r_{1}^{\left(b_{1}\right)}(x) d_{2}^{+}\left(b_{1}-x\right) \mathrm{d} x, \\
\vartheta_{3}^{+} & =\int_{x=0}^{b_{1}} r_{1}^{\left(b_{1}\right)}(x) d_{3}^{+}\left(b_{1}-x\right) \mathrm{d} x, \\
\vartheta_{4, i}^{+} & =\int_{x=0}^{b_{1}} \int_{y=0}^{x} r_{1}^{\left(b_{1}\right)}\left(b_{1}-y\right) g_{1 i}^{+}(x-y) \mathrm{d} y d_{4, i}^{+}(x) \mathrm{d} x, \quad i=1,2, \\
\vartheta_{5}^{+} & =\int_{x=0}^{b_{1}} r_{1}^{\left(b_{1}\right)}\left(b_{1}-x\right) d_{42}^{+}(x) \mathrm{d} x, \\
\vartheta_{6, i}^{+} & =\int_{x=0}^{b_{1}} \int_{y=0}^{x} r_{1}^{\left(b_{1}\right)}\left(b_{1}-y\right) g_{1, i}^{+}(x-y) \mathrm{d} y d_{5 i}^{+}(x) \mathrm{d} x, \\
\vartheta_{7}^{+} & =\int_{x=0}^{b_{1}} r_{1}^{\left(b_{1}\right)}\left(b_{1}-x\right) d_{52}^{+}(x) \mathrm{d} x, \quad i=1,2, \\
\vartheta_{8, i}^{+} & =\int_{x=0}^{b_{1}} \int_{y=0}^{x} r_{1}^{\left(b_{1}\right)}\left(b_{1}-y\right) g_{1, i}^{+}(x-y) \mathrm{d} y d_{6 i}^{+}(x) \mathrm{d} x,
\end{aligned} \quad i=1,2,
$$

\begin{tabular}{|c|c|c|c|c|c|c|}
\hline & \multirow{2}{*}{ Claim arrival } & \multirow{2}{*}{ State transition } & \multicolumn{2}{|l|}{ After a claim } & \multicolumn{2}{|c|}{ After a dividend } \\
\hline & & & Surplus & State & Surplus & State \\
\hline$\vartheta_{1}^{+}$ & 11 & & Above $b_{1}$ & 1 & $b_{1}$ & 1 \\
\hline$\vartheta_{2}^{+}$ & 12 & & Above $b_{2}$ & 2 & $b_{2}$ & 2 \\
\hline$\vartheta_{3}^{+}$ & 12 & & Between $b_{1}$ and $b_{2}$ & 2 & $\begin{array}{r}b_{2} \\
\text { or } b_{1}\end{array}$ & $\begin{array}{l}2 \\
1\end{array}$ \\
\hline$\vartheta_{4}^{+}$ & $1 i, i=1,2$ & & Below $b_{1}$ & $i$ & $b_{1}$ & 1 \\
\hline$\vartheta_{5}^{+}$ & & To 2 & Below $b_{1}$ & 2 & $b_{1}$ & 1 \\
\hline$\vartheta_{6}^{+}$ & $1 i, i=1,2$ & & Below $b_{1}$ & $i$ & $b_{2}$ & 2 \\
\hline$\vartheta_{7}^{+}$ & & To 2 & Below $b_{1}$ & 2 & $b_{2}$ & 2 \\
\hline$\vartheta_{8}^{+}$ & $1 i, i=1,2$ & & Below $b_{1}$ & $i$ & $\begin{array}{r}b_{2} \\
\text { or } b_{1}\end{array}$ & $\begin{array}{l}2 \\
1\end{array}$ \\
\hline$\vartheta_{9}^{+}$ & & To 2 & Below $b_{1}$ & 2 & $\begin{array}{r}b_{2} \\
\text { or } b_{1}\end{array}$ & $\begin{array}{l}2 \\
1\end{array}$ \\
\hline
\end{tabular}

In Table 2 we consider all the cases corresponding to $\vartheta_{k}^{+}, k=1, \ldots, 9$, according to the state transition or claim arrival at time $\mathcal{E}_{1}$, the surplus and state after that transition, and the surplus and state after dividend payment.

TABle 2. 
For $R_{\boldsymbol{b}}(0)=b_{1}$ and $J(0)=1$, define the following expected discounted times to reach one of the barriers after $\varepsilon_{1}$ :

$$
\begin{aligned}
& \rho_{1}=\int_{x=0}^{b_{1}} r_{1}^{\left(b_{1}\right)}(x)\left(1-G_{11}^{+}(x)\right) \mathrm{d} x, \\
& \rho_{2}=\int_{x=0}^{b_{1}} r_{1}^{\left(b_{1}\right)}(x)\left(1-G_{12}^{+}\left(x+b_{2}-b_{1}\right)\right) \mathrm{d} x, \\
& \rho_{3}=\int_{x=0}^{b_{1}} r_{1}^{\left(b_{1}\right)}(x) \int_{z=0}^{b_{2}-b_{1}} g_{12}^{+}(x+z) L\left(b_{1}+z\right) \mathrm{d} z \mathrm{~d} x, \\
& \rho_{4}=\int_{x=0}^{b_{1}} r_{1}^{\left(b_{1}\right)}(x) \int_{z=0}^{b_{2}-b_{1}} g_{12}^{+}(x+z) \tilde{v}_{22}^{\left(b_{1}, b_{2}\right)+}\left(b_{1}+z, \cdot\right) \mathrm{d} z \mathrm{~d} x, \\
& \rho_{5}=\int_{x=0}^{b_{1}} r_{1}^{\left(b_{1}\right)}(x) \int_{z=0}^{b_{2}-b_{1}} g_{12}^{+}(x+z) \tilde{v}_{22}^{\left(b_{1}, b_{2}\right)-}\left(b_{1}+z\right) \mathrm{d} z \mathrm{~d} x, \\
& \rho_{6, i}=\int_{x=0}^{b_{1}} \int_{y=0}^{x} r_{1}^{\left(b_{1}\right)}\left(b_{1}-y\right) g_{1 i}^{+}(x-y) \mathrm{d} y v_{i 1}^{+}\left(x, \cdot, b_{1}\right) \mathrm{d} x, \quad i=1,2, \\
& \rho_{7}=\int_{x=0}^{b_{1}} r_{1}^{\left(b_{1}\right)}\left(b_{1}-x\right) v_{21}^{+}\left(x, \cdot, b_{1}\right) \mathrm{d} x, \\
& \rho_{8, i}=\int_{x=0}^{b_{1}} \int_{y=0}^{x} r_{1}^{\left.b_{1}\right)}\left(b_{1}-y\right) g_{1 i}^{+}(x-y) \mathrm{d} y \int_{z=b_{2}-b_{1}}^{\infty} v_{i 2}^{+}\left(x, z, b_{1}\right) \mathrm{d} z \mathrm{~d} x, \quad i=1,2, \\
& \rho_{9}=\int_{x=0}^{b_{1}} r_{1}^{\left(b_{1}\right)}\left(b_{1}-x\right) \int_{z=b_{2}-b_{1}}^{\infty} v_{22}^{+}\left(x, z, b_{1}\right) \mathrm{d} z \mathrm{~d} x, \quad i=1,2, \\
& \rho_{10, i}=\int_{x=0}^{b_{1}} \int_{y=0}^{x} r_{1}^{\left(b_{1}\right)}\left(b_{1}-y\right) g_{1 i}^{+}(x-y) \mathrm{d} y \\
& \times \int_{z=0}^{b_{2}-b_{1}} v_{i 2}^{+}\left(x, z, b_{1}\right) L\left(b_{1}+z\right) \mathrm{d} z \mathrm{~d} x, \quad i=1,2, \\
& \rho_{11}=\int_{x=0}^{b_{1}} r_{1}^{\left(b_{1}\right)}\left(b_{1}-x\right) \int_{z=0}^{b_{2}-b_{1}} v_{22}^{+}\left(x, z, b_{1}\right) L\left(b_{1}+z\right) \mathrm{d} z \mathrm{~d} x, \\
& \rho_{12, i}=\int_{x=0}^{b_{1}} \int_{y=0}^{x} r_{1}^{\left(b_{1}\right)}\left(b_{1}-y\right) g_{1 i}^{+}(x-y) \mathrm{d} y \\
& \times \int_{z=0}^{b_{2}-b_{1}} v_{i 2}^{+}\left(x, z, b_{1}\right) \tilde{v}_{22}^{\left(b_{1}, b_{2}\right)+}\left(b_{1}+z, \cdot\right) \mathrm{d} z \mathrm{~d} x, \quad i=1,2, \\
& \rho_{13}=\int_{x=0}^{b_{1}} r_{1}^{\left(b_{1}\right)}\left(b_{1}-x\right) \int_{z=0}^{b_{2}-b_{1}} v_{22}^{+}\left(x, z, b_{1}\right) \tilde{v}_{22}^{\left(b_{1}, b_{2}\right)+}\left(b_{1}+z, \cdot\right) \mathrm{d} z \mathrm{~d} x, \quad i=1,2, \\
& \rho_{14, i}=\int_{x=0}^{b_{1}} \int_{y=0}^{x} r_{1}^{\left(b_{1}\right)}\left(b_{1}-y\right) g_{1 i}^{+}(x-y) \mathrm{d} y \\
& \times\left(\int_{z=0}^{b_{2}-b_{1}} v_{i 2}^{+}\left(x, z, b_{1}\right) \tilde{v}_{22}^{\left(b_{1}, b_{2}\right)-}\left(b_{1}+z\right) \mathrm{d} z+v_{i 2}^{+}\left(x, 0, b_{1}\right)\right) \mathrm{d} x, \quad i=1,2, \\
& \rho_{15}=\int_{x=0}^{b_{1}} r_{1}^{\left(b_{1}\right)}\left(b_{1}-x\right) \\
& \times\left(\int_{z=0}^{b_{2}-b_{1}} v_{22}^{+}\left(x, z, b_{1}\right) \tilde{v}_{22}^{\left(b_{1}, b_{2}\right)-}\left(b_{1}+z\right) \mathrm{d} z+v_{22}^{+}\left(x, 0, b_{1}\right)\right) \mathrm{d} x, \quad i=1,2 .
\end{aligned}
$$


TABle 3.

\begin{tabular}{|c|c|c|c|c|c|c|}
\hline & \multirow{2}{*}{ Claim arrival } & \multirow{2}{*}{ State transition } & \multicolumn{2}{|l|}{ After a claim } & \multicolumn{2}{|c|}{ Dividend } \\
\hline & & & Surplus & State & Barrier & State \\
\hline$\rho_{1}$ & 11 & & Above $b_{1}$ & 1 & $b_{1}$ & 1 \\
\hline$\rho_{2}$ & 12 & & Above $b_{2}$ & 2 & $b_{2}$ & 2 \\
\hline$\rho_{3}$ & 12 & & Between $b_{1}$ and $b_{2}$ & 2 & $b_{1}$ & 1 \\
\hline$\rho_{4}$ & 12 & & Between $b_{1}$ and $b_{2}$ & 2 & $b_{2}$ & 2 \\
\hline$\rho_{5}$ & 12 & & Between $b_{1}$ and $b_{2}$ & 2 & $b_{1}$ & 2 \\
\hline$\rho_{6, i}$ & $1 i, i=1,2$ & & Below $b_{1}$ & $i$ & $b_{1}$ & 1 \\
\hline$\rho_{7}$ & & To 2 & Below $b_{1}$ & 2 & $b_{1}$ & 1 \\
\hline$\rho_{8, i}$ & $1 i, i=1,2$ & & Below $b_{1}$ & $i$ & $b_{2}$ & 2 \\
\hline$\rho_{9}$ & & To 2 & Below $b_{1}$ & 2 & $b_{2}$ & 2 \\
\hline$\rho_{10, i}$ & $1 i, i=1,2$ & & Below $b_{1}$ & $i$ & $b_{1}$ & 1 \\
\hline$\rho_{11}$ & & To 2 & Below $b_{1}$ & 2 & $b_{1}$ & 1 \\
\hline$\rho_{12, i}$ & $1 i, i=1,2$ & & Below $b_{1}$ & $i$ & $b_{2}$ & 2 \\
\hline$\rho_{13}$ & & To 2 & Below $b_{1}$ & 2 & $b_{2}$ & 2 \\
\hline$\rho_{14, i}$ & $1 i, i=1,2$ & & Below $b_{1}$ & $i$ & $b_{1}$ & 2 \\
\hline$\rho_{15}$ & & To 2 & Below $b_{1}$ & 2 & $b_{1}$ & 2 \\
\hline
\end{tabular}

In Table 3 we consider all the cases corresponding to $\rho_{k}, k=1, \ldots, 15$, according to the state transition or claim arrival at time $\mathcal{E}_{2}$, the surplus and state after that transition, the dividend barrier reached, and the state when the dividend barrier is reached.

For $R_{\boldsymbol{b}}(0)=b_{1}$ and $J(0)=2$, define the following expected discounted dividend payments:

$$
\begin{aligned}
& v_{1}=\int_{x=0}^{b_{1}} \int_{y=0}^{x} \tilde{u}_{21}^{\left(0, b_{2}\right)+}\left(b_{1}, y\right) g_{21}^{+}(x-y) \mathrm{d} y\left(d_{4,1}^{+}(x)+d_{5,1}^{+}(x)+d_{6,1}^{+}(x)\right) \mathrm{d} x, \\
& v_{2}=\int_{x=0}^{b_{1}} \tilde{u}_{21}^{\left(0, b_{2}\right)+}\left(b_{1}, x\right)\left(d_{4,1}^{+}(x)+d_{5,1}^{+}(x)+d_{6,1}^{+}(x)\right) \mathrm{d} x, \\
& v_{3}=\int_{x=b_{1}}^{\infty} \int_{y=0}^{x \wedge b_{2}} \tilde{u}_{21}^{\left(0, b_{2}\right)+}\left(b_{1}, y\right) g_{21}^{+}(x-y) \mathrm{d} y\left(x-b_{1}\right) \mathrm{d} x, \\
& v_{4}=\int_{x=b_{1}}^{b_{2}} \tilde{u}_{21}^{\left(0, b_{2}\right)+}\left(b_{1}, x\right)\left(x-b_{1}\right) \mathrm{d} x,
\end{aligned}
$$

where $v_{1}$ and $v_{2}$ describe the discounted dividends when a state change occurs at $\varepsilon_{2}$ when the reserve is below $b_{1}$, and $v_{3}$ and $v_{4}$ describe the discounted dividends when a state change occurs when the reserve is above $b_{1}$.

Now consider the following discounted exit times:

$$
\begin{aligned}
C_{1} & =\int_{x=0}^{b_{1}} \int_{y=0}^{x} \tilde{u}_{21}^{\left(0, b_{2}\right)+}\left(b_{1}, y\right) g_{21}^{+}(x-y) \mathrm{d} y v_{1,1}^{+}\left(x, \cdot, b_{1}\right) \mathrm{d} x, \\
C_{2} & =\int_{x=0}^{b_{1}} \tilde{u}_{21}^{\left(0, b_{2}\right)+}\left(b_{1}, x\right) v_{1,1}^{+}\left(x, \cdot, b_{1}\right) \mathrm{d} x, \\
C_{3} & =\int_{x=b_{1}}^{\infty} \int_{y=0}^{x \wedge b_{2}} \tilde{u}_{21}^{\left(0, b_{2}\right)+}\left(b_{1}, y\right) g_{21}^{+}(x-y) \mathrm{d} y \mathrm{~d} x, \\
C_{4} & =\int_{x=b_{1}}^{b_{2}} \tilde{u}_{21}^{\left(0, b_{2}\right)+}\left(b_{1}, x\right) \mathrm{d} x,
\end{aligned}
$$


TABle 4.

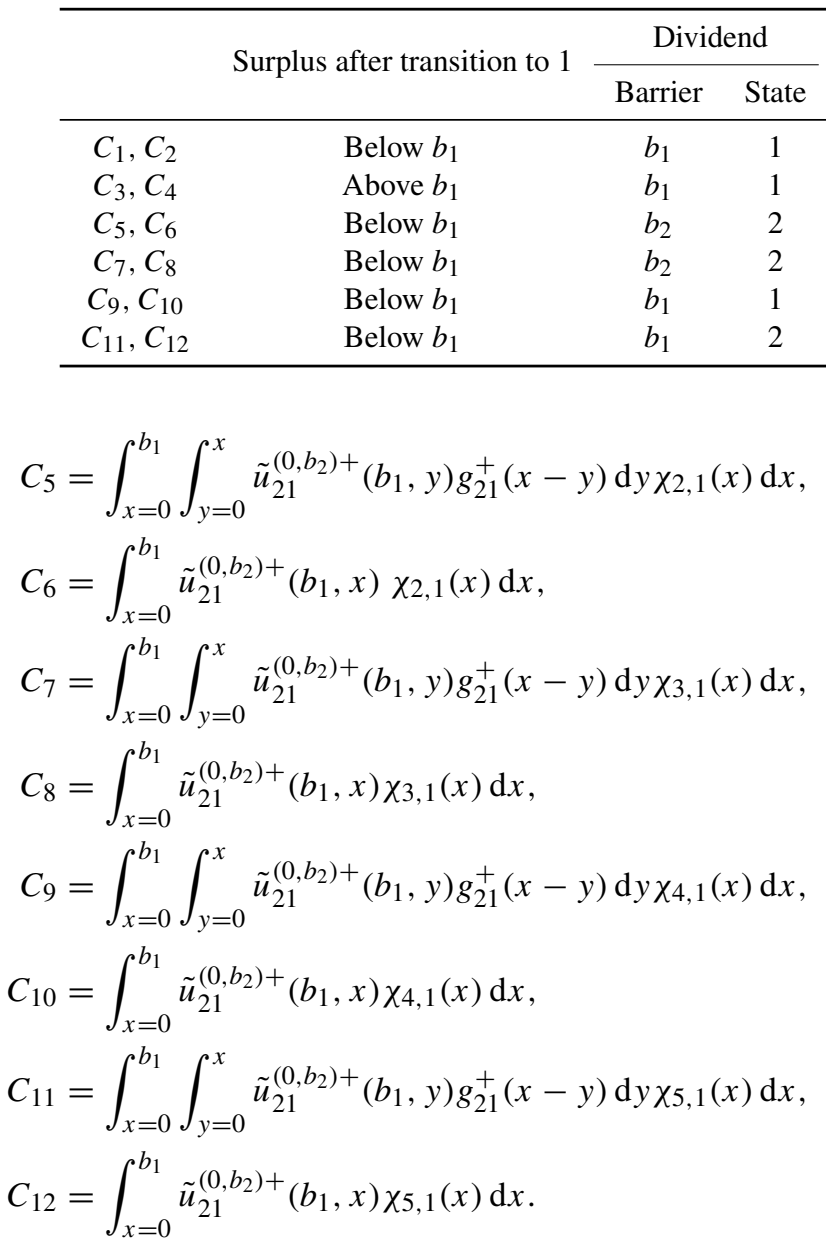

In Table 4 we summarize the process level after a transition to state 1 , the dividend barrier reached, and the state when the dividend barrier is reached.

Assume that $R_{\boldsymbol{b}}(0)=b_{2}$ and that $J(0)=2$. In this case we consider the process until it reaches one of the barriers $b_{i}, i=1,2$. Define now the expected discounted dividends paid until the process reaches one of the barriers:

$$
\begin{aligned}
& \varrho_{1}=\int_{x=0}^{b_{2}-b_{1}} r_{2}^{\left(b_{2}-b_{1}\right)}(x) \int_{z=0}^{\infty} g_{22}^{+}(x+z) z \mathrm{~d} z \mathrm{~d} x, \\
& \varrho_{2}=\int_{x=b_{1}}^{\infty} \int_{y=b_{1}}^{x \wedge b_{2}} r_{2}^{\left(b_{2}-b_{1}\right)}\left(b_{2}-y\right) g_{21}^{+}(x-y)\left(x-b_{1}\right) \mathrm{d} y \mathrm{~d} x, \\
& \varrho_{3}=\int_{x=b_{1}}^{b_{2}} r_{2}^{\left(b_{2}-b_{1}\right)}\left(b_{2}-x\right)\left(x-b_{1}\right) \mathrm{d} x, \\
& \varrho_{4}=\int_{x=b_{1}}^{b_{2}} \int_{y=b_{1}}^{x} r_{2}^{\left(b_{2}-b_{1}\right)}\left(b_{2}-y\right) g_{22}^{+}(x-y) \ell(x) \mathrm{d} y \mathrm{~d} x .
\end{aligned}
$$


Here $\varrho_{2}$ and $\varrho_{3}$ are the expected discounted dividends when at $\varepsilon_{2}$ there is a transition to state 1 , and $\varrho_{1}$ and $\varrho_{4}$ are the expected discounted dividends when at $\varepsilon_{2}$ there is a type-22 claim. Define the following expected discounted times to reach one of the barriers after $\varepsilon_{2}$ :

$$
\begin{aligned}
U_{1} & =\int_{x=0}^{b_{2}-b_{1}} r_{2}^{\left(b_{2}-b_{1}\right)}(x)\left(1-G_{22}^{+}(x)\right) \mathrm{d} x, \\
U_{2} & =\int_{x=0}^{b_{2}-b_{1}} r_{2}^{\left(b_{2}-b_{1}\right)}(x) \mathrm{d} x, \\
U_{3} & =\int_{x=b_{1}}^{b_{2}} \int_{y=b_{1}}^{x} r_{2}^{\left(b_{2}-b_{1}\right)}\left(b_{2}-y\right) g_{22}^{+}(x-y) L(x) \mathrm{d} y \mathrm{~d} x, \\
U_{4} & =\int_{x=b_{1}}^{b_{2}} \int_{y=b_{1}}^{x} r_{2}^{\left(b_{2}-b_{1}\right)}\left(b_{2}-y\right) g_{22}^{+}(x-y) \tilde{v}_{22}^{\left(b_{1}, b_{2}\right)+}(x, \cdot) \mathrm{d} y \mathrm{~d} x, \\
U_{5} & =\int_{x=b_{1}}^{b_{2}} \int_{y=b_{1}}^{x} r_{2}^{\left(b_{2}-b_{1}\right)}\left(b_{2}-y\right) g_{22}^{+}(x-y) \tilde{v}_{22}^{\left(b_{1}, b_{2}\right)-}(x) \mathrm{d} y \mathrm{~d} x .
\end{aligned}
$$

Here $U_{1}$ and $U_{4}$ are related to the expected discounted time to reach level $b_{2}$ when the modulated state is $2, U_{2}$ and $U_{3}$ are related to the expected discounted time to reach level $b_{1}$ when the modulated state is 1 , while $U_{5}$ is related to the expected discounted time to reach level $b_{1}$ when the modulated state is 2 .

Let $w_{2}^{\left(b_{2}-b_{1}\right)}$ be the expected discounted dividends paid until $\varepsilon_{2} \wedge T_{b_{2}-b_{1}, 2}$. It is obtained similarly to $w_{2}$ in (3.3) or (3.4) with $b_{2}-b_{1}$ replacing $b$. We are now able to derive linear equations for $V_{1}\left(b_{1}, \boldsymbol{b}\right), V_{2}\left(b_{1}, \boldsymbol{b}\right)$, and $V_{2}\left(b_{2}, \boldsymbol{b}\right)$.

Proposition 7.2. We have

$$
\begin{aligned}
V_{1}\left(b_{1}, \boldsymbol{b}\right)= & w_{1}^{\left(b_{1}\right)}+D_{1}(1,1)\left(\vartheta_{1}^{+}+\vartheta_{4,1}^{+}+\vartheta_{6,1}^{+}+\vartheta_{8,1}^{+}\right) \\
& +D_{1}(1,2)\left(\vartheta_{2}^{+}+\vartheta_{3}^{+}+\vartheta_{4,2}^{+}+\vartheta_{6,2}^{+}+\vartheta_{8,2}^{+}\right) \\
& +D_{0}(1,2)\left(\vartheta_{5}^{+}+\vartheta_{7}^{+}+\vartheta_{9}^{+}\right) \\
& +\left(D_{1}(1,1)\left(\rho_{1}+\rho_{6,1}+\rho_{10,1}\right)\right. \\
& \left.\quad+D_{1}(1,2)\left(\rho_{3}+\rho_{6,2}+\rho_{10,2}\right)+D_{0}(1,2)\left(\rho_{7}+\rho_{11}\right)\right) V_{1}\left(b_{1}, \boldsymbol{b}\right) \\
& +\left(D_{1}(1,2)\left(\rho_{5}+\rho_{14,2}\right)+D_{0}(1,2) \rho_{15}+D_{1}(1,1) \rho_{14,2}\right) V_{2}\left(b_{1}, \boldsymbol{b}\right) \\
& +\left(D_{1}(1,1)\left(\rho_{8,1}+\rho_{12,1}\right)+D_{1}(1,2)\left(\rho_{8,2}+\rho_{12,2}+\rho_{4}+\rho_{2}\right)\right. \\
& \left.+D_{0}(1,2)\left(\rho_{9}+\rho_{13}\right)\right) V_{2}\left(b_{2}, \boldsymbol{b}\right), \\
V_{2}\left(b_{1}, \boldsymbol{b}\right)= & D_{1}(2,1)\left(v_{1}+v_{3}\right)+D_{0}(2,1)\left(v_{2}+v_{4}\right) \\
& +\left(D_{1}(2,1)\left(C_{1}+C_{3}+C_{9}\right)+D_{0}(1,2)\left(C_{2}+C_{4}+C_{10}\right)\right) V_{1}\left(b_{1}, \boldsymbol{b}\right) \\
& +\left(D_{1}(1,2)\left(C_{5}+C_{7}\right)+D_{0}(1,2)\left(C_{6}+C_{8}\right)\right) V_{2}\left(b_{2}, \boldsymbol{b}\right) \\
& +\left(D_{1}(1,2) C_{11}+D_{0}(1,2) C_{12}\right) V_{2}\left(b_{1}, \boldsymbol{b}\right), \\
V_{2}\left(b_{2}, \boldsymbol{b}\right)= & w_{2}^{\left(b_{2}-b_{1}\right)}+D_{1}(2,2)\left(\varrho_{1}+\varrho_{4}\right)+D_{1}(2,1) \varrho_{2}+D_{0}(2,1) \varrho_{3} \\
& +\left(D_{0}(2,1) U_{2}+D_{1}(2,2) U_{3}\right) V_{1}\left(b_{1}, \boldsymbol{b}\right) \\
& +D_{1}(2,2) U_{5} V_{2}\left(b_{1}, \boldsymbol{b}\right)+D_{1}(2,2)\left(U_{1}+U_{4}\right) V_{2}\left(b_{2}, \boldsymbol{b}\right) .
\end{aligned}
$$

The proof of the proposition straightforwardly follows from the definitions of the coefficients. For $0<u<b_{1}, i=1,2$,

$$
\begin{aligned}
V_{i}(u, \boldsymbol{b})= & d_{4, i}^{+}(u)+d_{5, i}^{+}(u)+d_{6, i}^{+}(u)+\left(v_{i, 1}^{+}\left(u, \cdot, b_{1}\right)+\chi_{4, i}(u)\right) V_{1}\left(b_{1}, \boldsymbol{b}\right) \\
& +\left(\chi_{2, i}+\chi_{3, i}\right) V_{2}\left(b_{2}, \boldsymbol{b}\right)+\chi_{5, i}(u) V_{2}\left(b_{1}, \boldsymbol{b}\right) .
\end{aligned}
$$


For $b_{1}<u<b_{2}$,

$$
V_{2}(u, \boldsymbol{b})=\ell(u)+L(u) V_{1}\left(b_{1}, \boldsymbol{b}\right)+\tilde{v}_{22}^{\left(b_{1}, b_{2}\right)-}(u) V_{2}\left(b_{1}, \boldsymbol{b}\right)+\tilde{v}_{22}^{\left(b_{1}, b_{2}\right)-}(u, \cdot) V_{2}\left(b_{2}, \boldsymbol{b}\right) .
$$

Clearly, for $u>b_{i}$,

$$
V_{i}(u, b)=u-b_{i}+V_{i}\left(b_{i}, \boldsymbol{b}\right) .
$$

In the case of positive jumps the deficit at ruin is 0 . For the expected discounted time to ruin, we obtain similar equations, although easier since we do not consider overflows.

\section{References}

Ahn, S. And Badescu, A. L. (2007). On the analysis of the Gerber-Shiu discounted penalty function for risk processes with Markovian arrivals. Insurance Math. Econom. 41, 234-249.

Asmussen, S. (1989). Exponential families generated by phase-type distribution and other Markov lifetimes. Scand. J. Statist. 16, 319-334.

Asmussen, S. (2003). Applied Probability and Queues, 2nd edn. Springer, New York.

Avanzi, B. and Gerber, H. U. (2008). Optimal dividends in the dual model with diffusion. ASTIN Bull. 38, 653-667.

Avanzi, B., Gerber, H. U. AND ShIU, E. S. W. (2007). Optimal dividends in the dual model. Insurance Math. Econom. 41, 111-123.

Avram, F., Kyprianou, A. E. AND Pistorius, M. R. (2004). Exit problems for spectrally negative Lévy processes and applications to (Canadized) Russian options. Ann. Appl. Prob. 14, 215-238.

Bertoin, J. (1996). Lévy Processes. Cambridge University Press.

Breuer, L. (2008). First passage time for Markov additive processes with positive jumps of phase type. J. Appl. Prob. 45, 779-799.

Breuer, L. (2010) A quintuple law for Markov processes with phase-type jumps. J. Appl. Prob. 47, 441-458.

Cheung, E. C. K. (2011). On the class of stochastic models with two-sided jumps. Queueing Systems 69, 1-28.

Cheung, E. C. K. And Landriault, D. (2009). Perturbed MAP risk models with dividend barrier strategies. J. Appl. Prob. 46, 521-541.

Egami, M. And YamazaKi, K. (2012). Phase-type fitting of scale functions for spectrally negative Lévy processes. Preprint. Available at http://arxiv.org/abs/1005.0064v6.

Kyprianou, A. E. (2006), Introductory Lectures on Fluctuations of Lévy Processes with Applications. Springer, Berlin.

Kyprianou, A. E. ANd Palmowski, Z. (2008). Fluctuations of spectrally negative Markov additive processes. In Séminaire de Probabiltés XLI (Lecture Notes Math. 1934), Springer, Berlin, pp. 121-135.

LI, S. AND LU, Y. (2007). Moments of the dividend payments and related problems in a Markov-modulated risk model. N. Amer. Actuarial J. 11, 65-76.

LI, S. AND LU, Y. (2008). The decompositions of the discounted penalty functions and dividends-penalty identity in a Markov-modulated risk model. ASTIN Bull. 38, 53-71.

LU, Y. AND LI, S. (2009). The Markovian regime-switching risk model with threshold dividend strategy. Insurance Math. Econom. 44, 296-303.

Lu, Y. AND TsaI, C. C.-L. (2007). The expected discounted penalty at ruin for Markov-modulated risk process perturbed by diffusion. N. Amer. Actuarial J. 11, 136-149.

Pistorius, M. R. (2004). On exit and ergodicity of the spectrally one-sided Lévy process reflected at its infimum. J. Theoret. Prob. 17, 183-220.

ZHU, J. AND YANG, H. (2008). Ruin theory for a Markov regime-switching model under threshold dividend strategy. Insurance Math. Econom. 42, 311-318. 